Article

\title{
Palladium-Catalyzed Synthesis of Natural and Unnatural 2-, 5-, and 7-Oxygenated Carbazole Alkaloids from $N$-Arylcyclohexane Enaminones
}

\author{
Rafael Bautista, Pablo A. Montoya, Araceli Rebollar, Eleuterio Burgueño and Joaquín Tamariz * \\ Departamento de Química Orgánica, Escuela Nacional de Ciencias Biológicas, \\ Instituto Politécnico Nacional. Prol. Carpio y Plan de Ayala, México D.F. 11340, Mexico \\ * Author to whom correspondence should be addressed; E-Mail: jtamariz@woodward.encb.ipn.mx; \\ Tel.: +52-55-5729-6300 (ext. 62411); Fax: +52-55-5586-6621.
}

Received: 5 July 2013; in revised form: 5 August 2013 / Accepted: 12 August 2013 /

Published: 26 August 2013

\begin{abstract}
A palladium-catalyzed synthesis of the carbazole framework is described, including the preparation of 2-, 5-, and 7-oxygenated natural and unnatural carbazole alkaloids. A series of $N$-arylcyclohexane enaminones, generated by condensation of cyclohexane-1,3-dione with diverse anilines, were aromatized by a $\operatorname{Pd}(0)$-catalyzed thermal treatment to afford the corresponding diarylamines. The latter were submitted to a Pd(II)-catalyzed cyclization and methylation processes to provide the desired carbazoles, including clausine V. Following an inverse strategy, a new and short total synthesis of glycoborine is also reported.
\end{abstract}

Keywords: 2-oxygenated carbazoles; enaminones; palladium(II) cyclization; clausine V; glycoborine

\section{Introduction}

Biologically active carbazole alkaloids, a family of natural products with a variety of molecular structures, are isolated from higher order plants of the genera Clausena, Glycosmis, Micromelum, and Murraya (Rutaceae), among other sources [1-5]. Specifically, a great number of 2-, 5-, 6-, 7-mono- and bis-oxygenated tricyclic carbazoles isolated from these genera [1-3] exhibit a broad range of significant biological activities, including compounds with anti-tumor [6,7], antiplatelet aggregative [8], antibiotic [6,9,10], anti-viral [11-13], anti-plasmodial [14], anti-convulsant [15], 
and sigma receptor antagonist [16,17] properties. Carbazole derivatives $\mathbf{1 a}-\mathbf{g}$ are examples of these natural alkaloids $[8,18-23]$ (Figure 1).

Figure 1. Examples of naturally occurring 2-, 7-, and 2,7-oxygenated tricyclic carbazoles.

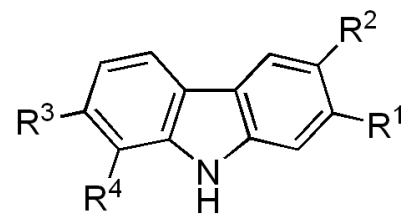

1a, Mukonal, $\mathrm{R}^{1}=\mathrm{OH}, \mathrm{R}^{2}=\mathrm{CHO}, \mathrm{R}^{3}=\mathrm{R}^{4}=\mathrm{H}$

1b, Clausine $A, R^{1}=O H, R^{2}=C H O, R^{3}=H, R^{4}=O M e$

1c, Murrayaline- $\mathrm{C}, \mathrm{R}^{1}=\mathrm{OH}, \mathrm{R}^{2}=\mathrm{R}^{4}=\mathrm{CHO}, \mathrm{R}^{3}=\mathrm{OMe}$

1d, Clausine $V, R^{1}=R^{3}=O M e, R^{2}=R^{4}=H$

1e, Clauszoline-C, $\mathrm{R}^{1}=\mathrm{R}^{3}=\mathrm{OMe}, \mathrm{R}^{2}=\mathrm{CO}_{2} \mathrm{Me}, \mathrm{R}^{4}=\mathrm{H}$

1f, Clauszoline-K, $\mathrm{R}^{1}=\mathrm{R}^{4}=\mathrm{H}, \mathrm{R}^{2}=\mathrm{CHO}, \mathrm{R}^{3}=\mathrm{OMe}$

1g, Clauszoline-L, $R^{1}=R^{4}=H, R^{2}=\mathrm{CO}_{2} \mathrm{Me}, \mathrm{R}^{3}=\mathrm{OMe}$

In spite of the large number of 2-, 5-, 6-, 7-mono- and bis-oxygenated tricyclic natural carbazoles that have been isolated, the wide range of functional groups and substitution patterns that exists among these compounds, and their important pharmacological activity, only recently a considerable number of synthetic approaches for their efficient preparation have been published [1-3,24-36].

We recently described a general synthetic approach for the construction of 1-methoxycarbazoles, including the naturally occurring alkaloid glycozolicine, which was accomplished with high overall yields through a three-step reaction sequence [37]. Based on this approach, we describe herein a new synthetic route for the preparation of 2-, 7-, and 2,7-oxygenated carbazoles 1. Starting from cyclohexene-1,3-dione (2) and the respective anilines 3a-e, enaminones $\mathbf{4 a - e}$ were prepared (Scheme 1). The latter were converted into diarylamines $\mathbf{5 a}-\mathbf{e}$ and then cyclized to the desired carbazoles $\mathbf{1}$, via an efficient Pd-catalyzed aromatization and cyclization sequence of reactions.

Scheme 1. Synthetic approach for the preparation of 2-oxygenated tricyclic carbazoles $\mathbf{1 .}$

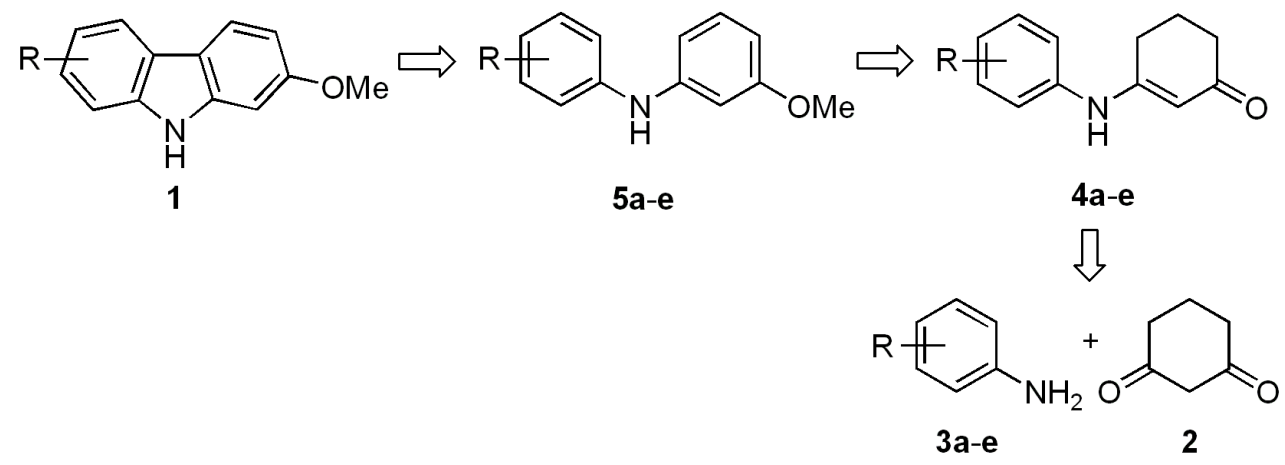

\section{Results and Discussion}

\subsection{Synthesis of Diarylamines}

The catalyst-free condensation of cyclohexane-1,3-dione (2) with anilines 3a-e provided 3-anilino2-cyclohexen-1-ones $\mathbf{4 a - e}$ in high yields (Table 1). However, the use of deactivated anilines, such as 
3-nitro- and 4-nitroanilines, failed to provide the desired enaminones, thus limiting this procedure to anilines substituted with electron-donating groups. Applying our previous procedure for aromatization using $\mathrm{Pd}(\mathrm{OAc})_{2}(30 \% \mathrm{~mol})$ [37], derivatives $\mathbf{4 a}-\mathbf{b}$ did not lead to the desired diarylamines $\mathbf{6 a}-\mathbf{b}$, but instead furnished the carbazole frame compounds $\mathbf{7 a - b}$ in good yields (Scheme 2). Similar results via Pd-mediated procedures have been reported for analogous substrates [1,38-41], which in turn have been transformed into the 4-oxygenated carbazoles [42]. When using other catalysts, such as mercuric acetate [43] and 2,3-dichloro-5,6-dicyano-p-benzoquinone (DDQ) [44,45], diarylamines 6 were indeed produced, but in very low yields $(15 \%-20 \%)$.

Table 1. Scope of the reaction between cyclohexane-1,3-dione (2) and anilines $\mathbf{3 a}-\mathbf{e}^{a}$.

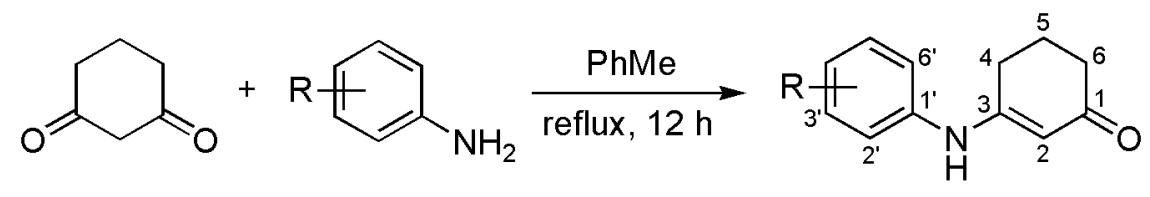

\begin{tabular}{ccc}
\multicolumn{2}{c}{ 3a-e } & 4a-e \\
\hline Entry & 3 (Ar) & $\mathbf{4}(\mathbf{\%})^{\boldsymbol{b}}$ \\
\hline 1 & $\mathbf{3 a}\left(\mathrm{C}_{6} \mathrm{H}_{4}-4-\mathrm{Me}\right)$ & $\mathbf{4 a}(92)$ \\
2 & $\mathbf{3 b}\left(\mathrm{C}_{6} \mathrm{H}_{4}-4-\mathrm{OMe}\right)$ & $\mathbf{4 b}(95)$ \\
3 & $\mathbf{3 c}\left(\mathrm{C}_{6} \mathrm{H}_{4}-3-\mathrm{Me}\right)$ & $\mathbf{4 c}(90)$ \\
4 & $\mathbf{3 d}\left(\mathrm{C}_{6} \mathrm{H}_{4}-3-\mathrm{OMe}\right)$ & $\mathbf{4 d}(93)$ \\
5 & $\mathbf{3 e}\left(\mathrm{C}_{6} \mathrm{H}_{3}-3,5-(\mathrm{OMe})_{2}\right.$ & $\mathbf{4 e}(96)$ \\
\hline
\end{tabular}

${ }^{a}$ Standard conditions: $2(3.57 \mathrm{mmol}), 3(3.57 \mathrm{mmol})$, toluene $(150 \mathrm{~mL})$, reflux, $12 \mathrm{~h}$.

${ }^{b}$ Isolated yields.

Scheme 2. Pd(II)-catalyzed treatment of 3-anilino-2-cyclohexen-1-ones $\mathbf{4 a - b}$.

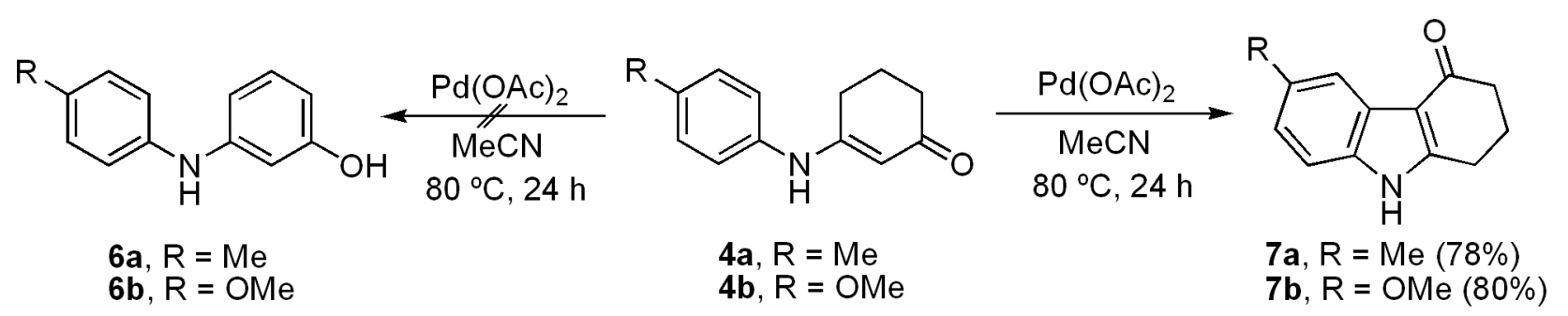

Due to the fact that the insertion of the aryl and cyclohexenone rings takes place via a $\operatorname{Pd}(\mathrm{II})$-catalyzed pathway $[1,2,38-41,46]$, we chose a $\operatorname{Pd}(0)$-mediated method for carrying out such an aromatization. Initially, when $\mathbf{4 b}$ was treated with $\mathrm{Pd} / \mathrm{C}(5 \%)$ at different concentrations (1-6 mol\%) with $\mathrm{MeOH}$ as the solvent and heating to $50-200{ }^{\circ} \mathrm{C}$ in a sealed vessel, diarylamine $\mathbf{6 b}$ was not obtained and the starting material was recovered. However, the desired transformation was achieved by increasing both the palladium(0) loading on charcoal $(10 \%)(1.9 \%-5.7 \% \mathrm{~mol})$ and the reaction temperature (Table 2, entries 1-3). The use of the $\operatorname{Pd}(0)$-mediated aromatization method for similar substrates or carbazole derivatives in moderate to good yields has been reported [42,47-52]. Reagents such as DDQ [44,45,53] and chloranil [54] have also been successfully applied to achieve analogous conversions $[43,55]$.

Although the preparation and purification of diarylamines $6 \mathbf{6}-\mathbf{b}$ and $\mathbf{6 d}-\mathbf{e}$ resulted in high yields (Table 2, entries 3-4 and 6-7), the relative instability of these compounds under the conditions of the 
following cyclization reaction made it necessary to protect the phenol moiety. In order to achieve this protection and taking into account that there are many naturally occurring methoxy-containing oxygenated carbazoles, we decided to obtain the methylated derivatives 5a-e. For this purpose, we employed a direct sequential procedure for the dehydrogenation and methylation of phenols 6 without purification (Table 2, entries 3-7). Thus, the series of compounds 5a-e was prepared in high yields $(81 \%-87 \%)$.

Table 2. Conversion of 3-anilino-2-cyclohexen-1-ones $4 \mathbf{a}-\mathbf{e}$ into diarylamines $\mathbf{6 a}-\mathbf{b}, \mathbf{6} \mathbf{d}-\mathbf{e}$ and $\mathbf{5 a}-\mathbf{e}^{a}$.

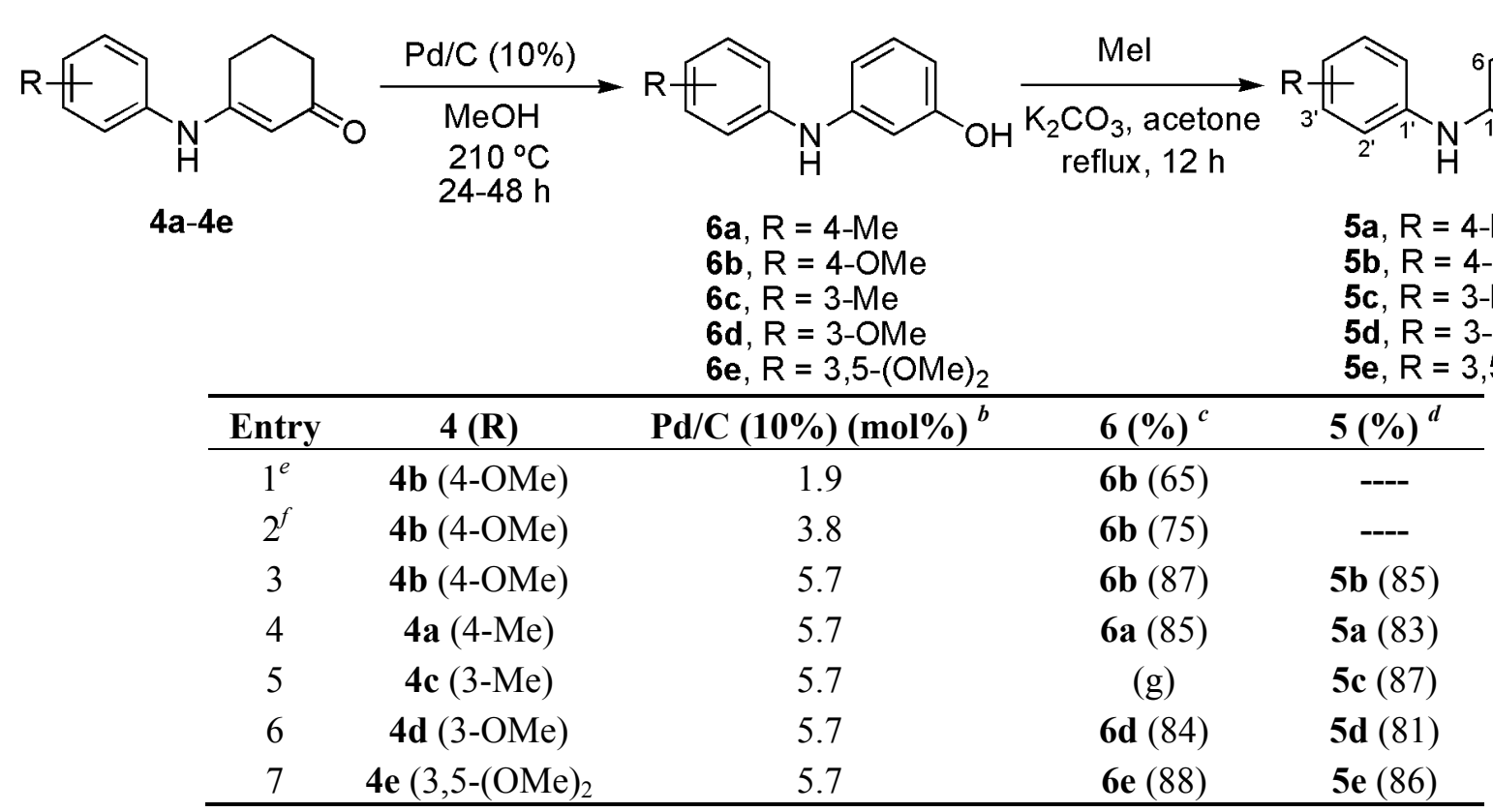

${ }^{a}$ Standard conditions: (a) Preparation of diarylamines 6: 4 (0.81-1.00 mmol), $\mathrm{Pd} / \mathrm{C}(10 \%), \mathrm{MeOH}, 210{ }^{\circ} \mathrm{C}$, $48 \mathrm{~h}$; (b) Preparation of diarylamines 5a-e: Aromatization step: 4 (0.82-1.00 mmol), $\mathrm{Pd} / \mathrm{C}(10 \%), \mathrm{MeOH}$, $210^{\circ} \mathrm{C}, 24 \mathrm{~h}$; Methylation step: 6 (1.0 mol equiv.), MeI (2.0 mol equiv.), $\mathrm{K}_{2} \mathrm{CO}_{3}$ (1.5 mol equiv.), acetone, reflux, 12 h. ${ }^{b}$ Calculated for $\operatorname{Pd}(0) .{ }^{c}$ Isolated yields. ${ }^{d}$ Isolated yields for the two steps. ${ }^{e}$ At $180{ }^{\circ} \mathrm{C}$ for $12 \mathrm{~h}$. ${ }^{f}$ At $200{ }^{\circ} \mathrm{C}$ for 48 h. ${ }^{g}$ Not isolated.

\subsection{Synthesis of Carbazoles}

The final cyclization step of diarylamines $5 \mathbf{a}-\mathbf{e}$ was successfully carried out by following the protocol originally developed by Knölker and coworkers [40,56,57], later applied by others [28,29], and optimized in our syntheses of natural carbazoles [37,44]. Thus, the conversion of the series $\mathbf{5 a - c}$ and $\mathbf{5 e}$ into the carbazole derivatives $\mathbf{1 h}-\mathbf{k}$ resulted in good yields (80\%-92\%) (Table 3). It is noteworthy that the cyclization of $\mathbf{5 d}$ provided clausine $\mathrm{V}$ (1d) in high yield $(90 \%)[22,33]$.

With the aim of testing the utility of this methodology for the total synthesis of natural 7-oxygenated tricyclic carbazoles, we carried out the conversion of derivative $\mathbf{1 h}$ into clauszoline-K (1f) and clauszoline-L (clausine C, 1g). Thus, upon applying the well-known procedure [58,59] for the synthesis of these [32] and other natural carbazoles [44], carbazole $\mathbf{1 h}$ was treated with DDQ in a mixture of $\mathrm{MeOH} / \mathrm{H}_{2} \mathrm{O}$ /acetone $(1: 1: 1)$ at room temperature for $45 \mathrm{~min}$ to give $\mathbf{1 f}$ in $70 \%$ yield (Scheme 3). 
Table 3. Preparation of carbazoles $\mathbf{1 d}$ and $\mathbf{1 h}-\mathbf{k}$ via $\mathrm{Pd}(\mathrm{II})$-catalyzed cyclization of diarylamines $\mathbf{5} \mathbf{a}-\mathbf{e}^{a}$.

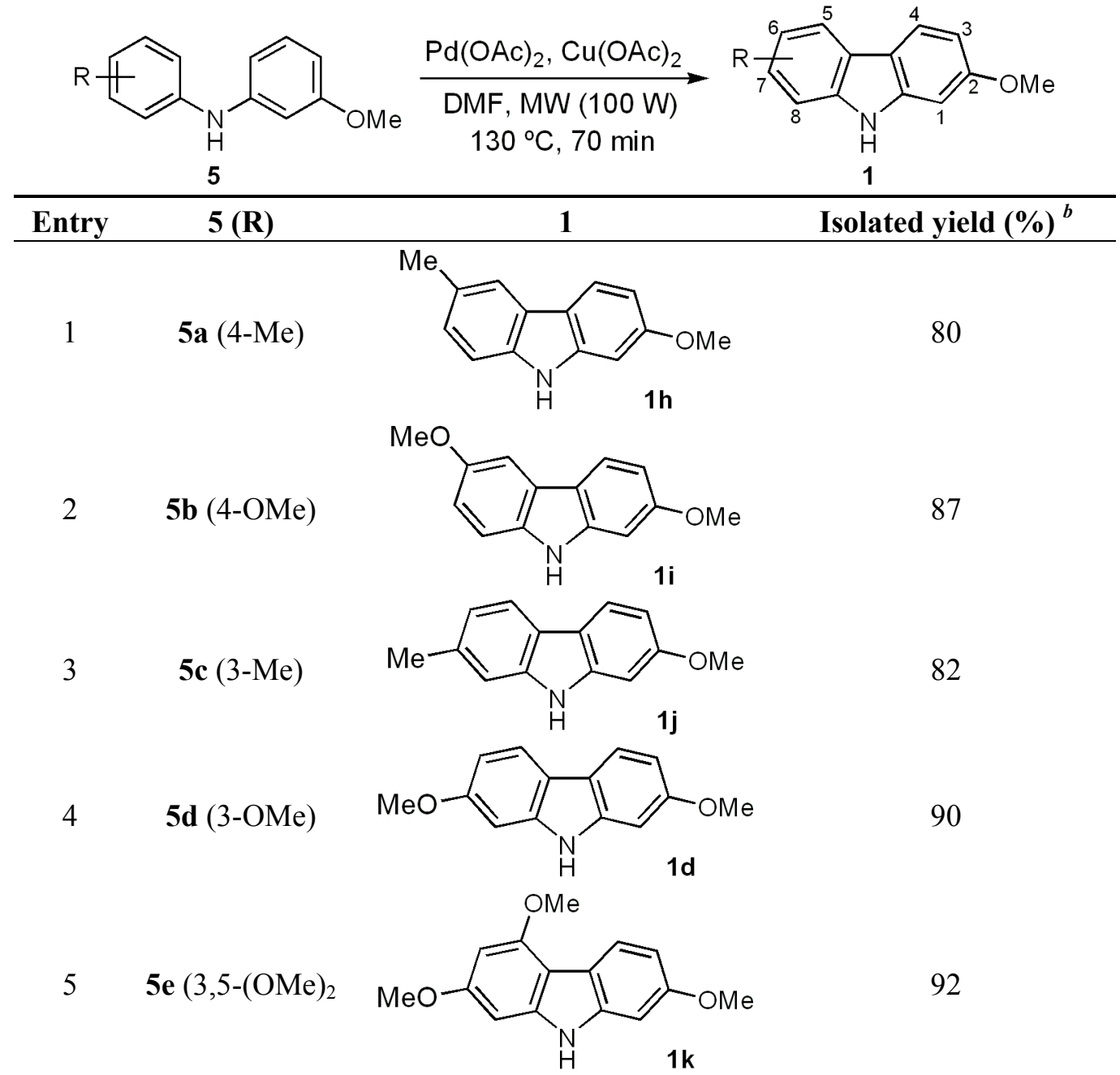

${ }^{a}$ Standard conditions: $5(0.32-0.47 \mathrm{mmol}), \mathrm{Pd}(\mathrm{OAc})_{2}(10 \mathrm{~mol} \%), \mathrm{Cu}(\mathrm{OAc})_{2}(2.5 \mathrm{~mol}$ equiv. $), \mathrm{DMF}, \mathrm{MW}$ $(100 \mathrm{~W}), 130{ }^{\circ} \mathrm{C}, 70 \mathrm{~min} .{ }^{b}$ Isolated yields.

Scheme 3. Preparation of natural carbazoles clauszoline-K (1f) and clauszoline-L (1g).<smiles>COc1ccc2c(c1)[nH]c1ccc(C=O)cc12</smiles><smiles>COc1ccc2c(c1)[nH]c1ccc(C(C)=O)cc12</smiles>

The latter was oxidized with a mixture of $\mathrm{MnO}_{2} / \mathrm{KCN}$ in $\mathrm{MeOH}$ [58] to furnish clauszoline-L (1g) in almost quantitative yield. The spectral data of the products obtained agree with those described for the natural [20,23] and synthetic [58] products. 


\subsection{Total Synthesis of Glycoborine (Glycrophylamine, 9)}

Recently, 5-methoxy-3-methylcarbazole (9) was isolated from the roots and branches of Glycosmis macrophylla and named glycrophylamine. This compound showed cytotoxic activity against NC1-H187 cancerigene cells [60]. However, the same carbazole had been isolated from Glycosmis arborea a decade earlier, and named glycoborine. This was the first 5-oxygenated tricyclic natural carbazole ever isolated [61]. Nowadays, three routes of synthesis have been developed for 9 based on Fischer [61], Japp-Klingemann [62], and Cadogan cyclizations [33] as the key step. We herein describe a new total synthesis of $\mathbf{9}$ starting from the key precursor tetrahydrocarbazole $\mathbf{7 a}$ (Scheme 4), which was efficiently prepared from $\mathbf{4 a}$ (Scheme 2$)$.

When a mixture of 7a, $\mathrm{Pd} / \mathrm{C}(10 \%)(5.7 \% \mathrm{~mol})$ and anhydrous $\mathrm{MeOH}$ was heated in a sealed vessel to $270{ }^{\circ} \mathrm{C}$ for $48 \mathrm{~h}$, 5-hydroxy-3-methylcarbazole (8) was isolated and then purified in good yield (Scheme 4). Methylation of the latter under the usual reaction conditions provided the desired natural carbazole 9, which was synthesized in four steps with high overall yield (53\%). The spectral data of 9 agree with those described for the natural $[60,61]$ and synthetic $[33,62]$ products.

Scheme 4. Preparation of natural carbazole glycoborine (9).

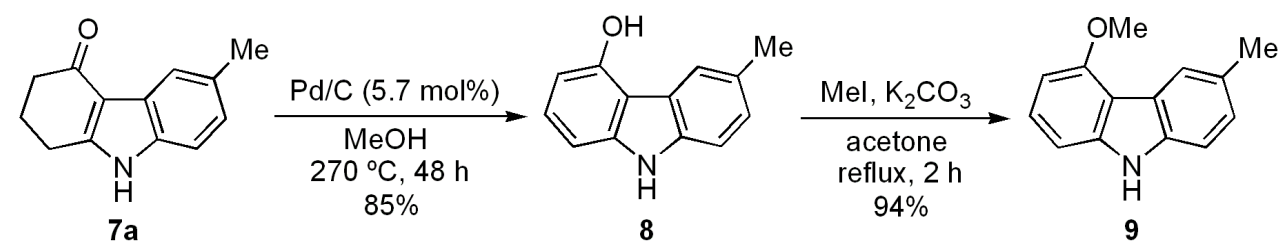

All the structures of intermediates and products described in these synthetic sequences were characterized by ${ }^{1} \mathrm{H}$ - and ${ }^{13} \mathrm{C}-\mathrm{NMR}$ spectroscopy, with the help of $2 \mathrm{D}$ (HMQC and HMBC) experiments and mass spectrometric techniques (MS and HRMS).

\section{Experimental}

\subsection{General}

Melting points (uncorrected) were determined with an Electrothermal capillary melting point apparatus. IR spectra were recorded on a Perkin-Elmer 2000 spectrophotometer. ${ }^{1} \mathrm{H}$ (300 or $500 \mathrm{MHz}$ ) and ${ }^{13} \mathrm{C}$-NMR (75 or $125 \mathrm{MHz}$ ) spectra were recorded on Varian Mercury-300 or Varian VNMR System instruments, with TMS as internal standard. Mass spectra (MS) and high-resolution mass spectra (HRMS) were obtained, in electron impact (EI) $(70 \mathrm{eV})$ mode, on Thermo-Finnigan Polaris Q and Jeol JSM-GcMateII spectrometers, respectively. Microwave (MW) irradiation was performed on a CEM MW reactor. Analytical thin-layer chromatography was carried out using E. Merck silica gel 60 $\mathrm{F}_{254}$ coated 0.25 plates, visualized by a long- and short-wavelength UV lamp. Flash column chromatography was performed over Natland International Co. silica gel (230-400 mesh). All air moisture sensitive reactions were carried out under nitrogen using oven-dried glassware. Toluene, $\mathrm{MeOH}$, and $\mathrm{MeCN}$ were freshly distilled over sodium and DMF over calcium hydride prior to use. Acetone was dried by distillation after treatment with potassium permanganate. $\mathrm{K}_{2} \mathrm{CO}_{3}$ was dried overnight at $200{ }^{\circ} \mathrm{C}$ prior to use. All other reagents were used without further purification. 


\subsection{Synthesis and Characterization}

3-(p-Tolylamino)cyclohex-2-en-1-one (4a) [63]. In a $250 \mathrm{~mL}$, three necked, round-bottomed flask equipped with a magnetic stirring bar, rubber septum, a water condenser and a Dean-Stark trap, under $\mathrm{N}_{2}$ atmosphere, a mixture of $2(0.400 \mathrm{~g}, 3.57 \mathrm{mmol})$ and $\mathbf{3 a}(0.382 \mathrm{~g}, 3.37 \mathrm{mmol})$ in dry toluene $(150 \mathrm{~mL})$ was stirred at reflux for $12 \mathrm{~h}$. The solvent was removed under vacuum, and the residue purified by column chromatography over silica gel (10 g/g of crude, hexane/EtOAc, 1:1) to give $4 \mathbf{a}(0.66 \mathrm{~g}, 92 \%)$ as a pale yellow solid. $R_{\mathrm{f}} 0.15$ (hexane/EtOAc, 1:1); mp 248-249 ${ }^{\circ} \mathrm{C}$. IR (KBr): $v_{\max } 3214,3029,2937$, $1573,1512,1361,1311,1245,1183,1141,818 \mathrm{~cm}^{-1} .{ }^{1} \mathrm{H}-\mathrm{NMR}\left(500 \mathrm{MHz}, \mathrm{CDCl}_{3}\right): \delta=1.98$ (qu, $J=6.5 \mathrm{~Hz}, 2 \mathrm{H}, \mathrm{H}-5), 2.31$ (br t, $J=6.5 \mathrm{~Hz}, 2 \mathrm{H}, \mathrm{H}-6), 2.32(\mathrm{~s}, 3 \mathrm{H}, \mathrm{CH}), 2.48$ (t, $J=6.5 \mathrm{~Hz}, 2 \mathrm{H}, \mathrm{H}-4)$, 5.48 (s, 1H, H-2), 6.99-7.03 (m, 2H, H-2'), 7.07-7.11 (m, 2H, H-3'), 7.12 (br s, $1 \mathrm{H}, \mathrm{NH}) .{ }^{13} \mathrm{C}-\mathrm{NMR}$ $\left(125 \mathrm{MHz} \mathrm{CDCl}_{3}\right): \delta=20.9\left(\mathrm{CH}_{3}\right), 21.8(\mathrm{C}-5), 29.5(\mathrm{C}-4), 36.4(\mathrm{C}-6), 99.0(\mathrm{C}-2), 124.0\left(\mathrm{C}-2^{\prime}\right), 129.7$ (C-3'), 135.3 (C-4'), 135.5 (C-1'), 163.1 (C-3), 198.1 (C-1). MS (70 eV): m/z (\%) $201\left(\mathrm{M}^{+}, 74\right), 184$

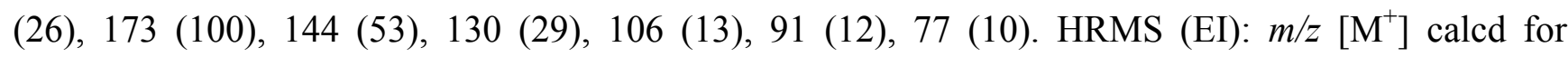
$\mathrm{C}_{13} \mathrm{H}_{15} \mathrm{NO}$ : 201.1154; found: 201.1156 .

3-(4-Methoxyphenylamino)cyclohex-2-en-1-one (4b). Following the procedure described for $\mathbf{4 a}$, using $2(0.400 \mathrm{~g}, 3.57 \mathrm{mmol})$ and $\mathbf{3 b}(0.439 \mathrm{~g}, 3.57 \mathrm{mmol}), \mathbf{4 b}(0.74 \mathrm{~g}, 95 \%)$ was obtained as a pale yellow solid. $R_{\mathrm{f}} 0.12$ (hexane/EtOAc, 1:1); mp $166-167{ }^{\circ} \mathrm{C}$ [Lit. [64] $164-166{ }^{\circ} \mathrm{C}$ ]. IR (KBr): $v_{\max } 3218$, 3039, 2946, 1513, 1412, 1365, 1243, 1180, 1135, 1032, 834, $716 \mathrm{~cm}^{-1} .{ }^{1} \mathrm{H}-\mathrm{NMR}\left(500 \mathrm{MHz}, \mathrm{CDCl}_{3}\right)$ : $\delta=1.97(\mathrm{qu}, J=6.5 \mathrm{~Hz}, 2 \mathrm{H}, \mathrm{H}-5), 2.30(\mathrm{t}, J=6.5 \mathrm{~Hz}, 2 \mathrm{H}, \mathrm{H}-6), 2.47$ (t, $J=6.5 \mathrm{~Hz}, 2 \mathrm{H}, \mathrm{H}-4), 3.78$ (s, $3 \mathrm{H}, \mathrm{CH}_{3} \mathrm{O}$ ), 5.34 (s, 1H, H-2), 6.80-6.84 (m, 2H, H-3'), 7.02-7.06 (m, 2H, H-2'), 7.13 (br s, 1H, NH). ${ }^{13} \mathrm{C}-\mathrm{NMR}\left(125 \mathrm{MHz}, \mathrm{CDCl}_{3}\right): \delta=21.8(\mathrm{C}-5), 29.3(\mathrm{C}-4), 36.4(\mathrm{C}-6), 55.4\left(\mathrm{CH}_{3} \mathrm{O}\right), 98.6(\mathrm{C}-2), 114.4$ (C-3'), 126.1 (C-2'), 130.8 (C-1'), 157.5 (C-4'), 164.0 (C-3), 198.0 (C-1). MS (70 eV): m/z (\%) 217 (M $\left.\mathrm{M}^{+}, 100\right), 200$ (55), 189 (43), 174 (20), 160 (98), 146 (30), 130 (23), 117 (12), 77 (10). HRMS (EI): $m / z\left[\mathrm{M}^{+}\right]$calcd for $\mathrm{C}_{13} \mathrm{H}_{15} \mathrm{NO}_{2}: 217.1103$; found: 217.1110 .

3-(m-Tolylamino)cyclohex-2-en-1-one (4c). Following the procedure described for $4 \mathbf{a}, 4 \mathbf{c}(0.65 \mathrm{~g}$, $90 \%)$ was obtained as a pale yellow oil from $2(0.400 \mathrm{~g}, 3.57 \mathrm{mmol})$ and $3 \mathrm{c}(0.382 \mathrm{~g}, 3.57 \mathrm{mmol})$. $R_{\mathrm{f}}$ 0.15 (hexane/EtOAc, 1:1). IR (film): $v_{\max } 3256,3066,2959,1546,1453,1360,1244,1185,1136,829$, 795, $728 \mathrm{~cm}^{-1} .{ }^{1} \mathrm{H}-\mathrm{NMR}\left(500 \mathrm{MHz}, \mathrm{CDCl}_{3}\right): \delta=1.98(\mathrm{qu}, J=6.5 \mathrm{~Hz}, 2 \mathrm{H}, \mathrm{H}-5), 2.29\left(\mathrm{~s}, 3 \mathrm{H}, \mathrm{CH}_{3}\right)$, 2.32 (t, $J=6.5 \mathrm{~Hz}, 2 \mathrm{H}, \mathrm{H}-6), 2.49$ (t, $J=6.5 \mathrm{~Hz}, 2 \mathrm{H}, \mathrm{H}-4), 5.55$ (s, 1H, H-2), 6.91-6.95 (m, 2H, H-4', H-6'), 6.96 (br s, $1 \mathrm{H}, \mathrm{H}-2^{\prime}$ ), 7.17 (t, $\left.J=8.0 \mathrm{~Hz}, 1 \mathrm{H}, \mathrm{H}-5^{\prime}\right), 7.18$ (br s, $\left.1 \mathrm{H}, \mathrm{NH}\right) .{ }^{13} \mathrm{C}-\mathrm{NMR}(125 \mathrm{MHz}$, $\left.\mathrm{CDCl}_{3}\right): \delta 21.3\left(\mathrm{CH}_{3}\right), 21.8(\mathrm{C}-5), 29.6(\mathrm{C}-4), 36.4(\mathrm{C}-6), 99.3(\mathrm{C}-2), 120.9\left(\mathrm{C}-6^{\prime}\right), 124.4\left(\mathrm{C}-2^{\prime}\right), 126.2$ (C-4'), 129.0 (C-5'), 138.0 (C-3'), 139.1 (C-1'), 162.9 (C-3), 198.3 (C-1). MS (70 eV): m/z (\%) 201 (M+, 79$), 184$ (49), 173 (100), 158 (16), 144 (88), 130 (42), 106 (13), 91 (19), 77 (16). HRMS (EI): $\mathrm{m} / z\left[\mathrm{M}^{+}\right]$calcd for $\mathrm{C}_{13} \mathrm{H}_{15} \mathrm{NO}: 201.1154$; found: 201.1160 .

3-(3-Methoxyphenylamino)cyclohex-2-en-1-one (4d). Following the procedure described for $\mathbf{4 a}$, using $2(0.400 \mathrm{~g}, 3.57 \mathrm{mmol})$ and $\mathbf{3 d}(0.439 \mathrm{~g}, 3.57 \mathrm{mmol}), \mathbf{4 d}(0.72 \mathrm{~g}, 93 \%)$ was obtained as a pale yellow solid. $R_{\mathrm{f}} 0.11$ (hexane/EtOAc, 1:1); mp 126-127 ${ }^{\circ} \mathrm{C}$ [Lit. [64] 122.5-124 ${ }^{\circ} \mathrm{C}$; [65] 126-128 ${ }^{\circ} \mathrm{C}$ ]. IR $(\mathrm{KBr}): v_{\max } 3278,3196,3133,2937,1540,1425,1357,1318,1245,1191,1143,1051,871,733 \mathrm{~cm}^{-1}$. ${ }^{1} \mathrm{H}-\mathrm{NMR}\left(500 \mathrm{MHz} \mathrm{CDCl}_{3}\right.$ ): $\delta 1.98(\mathrm{qu}, J=6.5 \mathrm{~Hz}, 2 \mathrm{H}, \mathrm{H}-5), 2.32(\mathrm{t}, J=6.5 \mathrm{~Hz}, 2 \mathrm{H}, \mathrm{H}-6), 2.50$ (t, 
$J=6.5 \mathrm{~Hz}, 2 \mathrm{H}, \mathrm{H}-4), 3.75\left(\mathrm{~s}, 3 \mathrm{H}, \mathrm{CH}_{3} \mathrm{O}\right), 5.59(\mathrm{~s}, 1 \mathrm{H}, \mathrm{H}-2), 6.66-6.70\left(\mathrm{~m}, 2 \mathrm{H}, \mathrm{H}-2^{\prime}, \mathrm{H}-6^{\prime}\right), 6.72$ (dm, $\left.J=8.0 \mathrm{~Hz}, 1 \mathrm{H}, \mathrm{H}-4^{\prime}\right), 7.16-7.21$ (m, $\left.1 \mathrm{H}, \mathrm{H}-5^{\prime}\right), 7.29$ (br s, $\left.1 \mathrm{H}, \mathrm{NH}\right) .{ }^{13} \mathrm{C}-\mathrm{NMR}\left(125 \mathrm{MHz}, \mathrm{CDCl}_{3}\right): \delta$ 21.8 (C-5), 29.5 (C-4), 36.4 (C-6), $55.2\left(\mathrm{CH}_{3} \mathrm{O}\right), 99.6$ (C-2), 109.7 (C-2'), $110.8\left(\mathrm{C}-6^{\prime}\right), 116.0\left(\mathrm{C}-4^{\prime}\right)$, 129.9 (C-5'), 139.4 (C-1'), 160.2 (C-3'), 162.7 (C-3), 198.4 (C-1). MS (70 eV): m/z (\%) $217\left(\mathrm{M}^{+}, 99\right)$, 200 (59), 189 (46), 160 (100), 146 (32), 130 (23), 117 (11), 77 (7). HRMS (EI): m/z [M $\left.{ }^{+}\right]$calcd for $\mathrm{C}_{13} \mathrm{H}_{15} \mathrm{NO}_{2}$ : 217.1103; found: 217.1101 .

3-(3,5-Dimethoxyphenylamino)cyclohex-2-en-1-one (4e). Following the procedure described for 4a, with $2(0.400 \mathrm{~g}, 3.57 \mathrm{mmol})$ and $3 \mathbf{e}(0.546 \mathrm{~g}, 3.57 \mathrm{mmol}), 4 \mathrm{e}(0.85 \mathrm{~g}, 96 \%)$ was obtained as a pale yellow solid. $R_{\mathrm{f}} 0.12$ (hexane/EtOAc, 1:1); mp 139-140 ${ }^{\circ} \mathrm{C}$. IR (KBr): $v_{\max } 3272,2940,1598,1582$, 1538, 1462, 1423, 1361, 1253, 1186, 1153, 1055, $824 \mathrm{~cm}^{-1} .{ }^{1} \mathrm{H}-\mathrm{NMR}\left(500 \mathrm{MHz}, \mathrm{CDCl}_{3}\right): \delta=1.99$ (qu, $J=6.5 \mathrm{~Hz}, 2 \mathrm{H}, \mathrm{H}-5), 2.33$ (t, $J=6.5 \mathrm{~Hz}, 2 \mathrm{H}, \mathrm{H}-6), 2.49$ (t, $J=6.5 \mathrm{~Hz}, 2 \mathrm{H}, \mathrm{H}-4), 3.73$ (s, 6H, $\left.2 \mathrm{CH}_{3} \mathrm{O}\right), 5.64(\mathrm{~s}, 1 \mathrm{H}, \mathrm{H}-2), 6.24\left(\mathrm{t}, J=2.0 \mathrm{~Hz}, 1 \mathrm{H}, \mathrm{H}-4^{\prime}\right), 6.30$ (d, $\left.J=2.0 \mathrm{~Hz}, 2 \mathrm{H}, \mathrm{H}-2^{\prime}, \mathrm{H}-6^{\prime}\right), 7.06$ (br s, $1 \mathrm{H}, \mathrm{NH}) .{ }^{13} \mathrm{C}-\mathrm{NMR}\left(125 \mathrm{MHz}, \mathrm{CDCl}_{3}\right): \delta=21.8(\mathrm{C}-5), 29.6(\mathrm{C}-4), 36.4(\mathrm{C}-6), 55.3\left(\mathrm{CH}_{3} \mathrm{O}\right)$, 97.4 (C-4'), 100.2 (C-2), 102.1 (C-2', C-6'), 139.9 (C-1'), 161.2 (C-3', C-5'), 162.3 (C-3), 198.3 (C-1). MS (70 eV): m/z (\%) 247 (M+37), 230 (100), 219 (25), 190 (83), 160 (18), 135 (30), 120 (14), 77 (7). HRMS (EI): $m / z$ [M $]$ calcd for $\mathrm{C}_{14} \mathrm{H}_{17} \mathrm{NO}_{3}: 247.1208$; found: 247.1207 .

3-(p-Tolylamino)phenol (6a). In a threaded ACE glass pressure tube with a sealed Teflon screw cap, under $\mathrm{N}_{2}$ atmosphere, a mixture of $4 \mathrm{a}(0.20 \mathrm{~g}, 1.0 \mathrm{mmol})$ and $\mathrm{Pd} / \mathrm{C}(10 \%)(0.060 \mathrm{~g}, 0.057 \mathrm{mmol})$ in dry $\mathrm{MeOH}(2.5 \mathrm{~mL})$ was stirred at $210{ }^{\circ} \mathrm{C}$ for $48 \mathrm{~h}$. The solvent was removed under vacuum, and the residue purified by column chromatography over silica gel (20 g/g of crude, hexane/EtOAc, 80:20), to give $6 \mathbf{6}(0.168 \mathrm{~g}, 85 \%)$ as a pale grey solid. $R_{\mathrm{f}} 0.55$ (hexane/EtOAc, 7:3); mp 81-82 ${ }^{\circ} \mathrm{C}$ [Lit. [66] $82{ }^{\circ} \mathrm{C}$ ]. IR (film): $v_{\max } 3394,1606,1512,1493,1332,1243,1155,969,815,766 \mathrm{~cm}^{-1}$. ${ }^{1} \mathrm{H}-\mathrm{NMR}(300 \mathrm{MHz}$, $\left.\mathrm{CDCl}_{3}\right): \delta=2.27\left(\mathrm{~s}, 3 \mathrm{H}, \mathrm{CH}_{3}\right), 5.58($ br s, $1 \mathrm{H}, \mathrm{NH}), 6.31(\mathrm{dd}, J=7.8,2.1 \mathrm{~Hz}, 1 \mathrm{H}, \mathrm{H}-4), 6.44(\mathrm{t}, J=2.1 \mathrm{~Hz}$, $1 \mathrm{H}, \mathrm{H}-2), 6.51$ (dd, $J=7.8,2.1 \mathrm{~Hz}, 1 \mathrm{H}, \mathrm{H}-6), 6.92-6.99$ (m, 2H, H-2'), 6.99-7.07 (m, 3H, H-3', $\mathrm{H}-5) .{ }^{13} \mathrm{C}-\mathrm{NMR}\left(75.4 \mathrm{MHz}, \mathrm{CDCl}_{3}\right): \delta=20.6\left(\mathrm{CH}_{3}\right), 103.2(\mathrm{C}-2), 107.1$ (C-4), $108.9(\mathrm{C}-6), 119.5$ $\left(\mathrm{C}-2^{\prime}\right), 129.8\left(\mathrm{C}-3^{\prime}\right), 130.2$ (C-5), $131.2\left(\mathrm{C}-4^{\prime}\right), 139.7$ (C-1'), $145.6(\mathrm{C}-1), 156.6$ (C-3). MS (70 eV): $m / z$ (\%) $199\left(\mathrm{M}^{+}, 100\right), 183$ (19), 170 (22), 154 (35), 128 (14), 91 (83), 65 (18). HRMS (EI): $m / z$ [M ${ }^{+}$] calcd for $\mathrm{C}_{13} \mathrm{H}_{13} \mathrm{NO}: 199.0997$; found: 199.0998 .

3-(4-Methoxyphenylamino)phenol (6b). Following the procedure described for $\mathbf{6 a}$, with $\mathbf{4 b}(0.200 \mathrm{~g}$, $0.92 \mathrm{mmol})$ and $\mathrm{Pd} / \mathrm{C}(10 \%)(0.060 \mathrm{~g}, 0.057 \mathrm{mmol}), 6 \mathbf{b}(0.172 \mathrm{~g}, 87 \%)$ was obtained as a pale grey solid. $R_{\mathrm{f}} 0.50$ (hexane/EtOAc, 8:2); mp 66-67 ${ }^{\circ} \mathrm{C}$ [Lit. [66] 67-68 ${ }^{\circ} \mathrm{C}$ ]. IR (KBr): $v_{\max } 3379,1601$, 1526, 1504, 1459, 1291, 1239, 1174, 1110, 1027, $735 \mathrm{~cm}^{-1} .{ }^{1} \mathrm{H}-\mathrm{NMR}\left(300 \mathrm{MHz}, \mathrm{CDCl}_{3}\right): \delta=3.79$ $\left(\mathrm{s}, 3 \mathrm{H}, \mathrm{CH}_{3} \mathrm{O}\right), 6.50$ (br s, $\left.1 \mathrm{H}, \mathrm{NH}\right), 6.28$ (ddd, $\left.J=8.0,2.4,0.6 \mathrm{~Hz}, 1 \mathrm{H}, \mathrm{H}-4\right), 6.37$ (t, $J=2.4 \mathrm{~Hz}, 1 \mathrm{H}$, H-2), 6.44 (ddd, $J=8.0,2.4,0.6 \mathrm{~Hz}, 1 \mathrm{H}, \mathrm{H}-6), 6.82-6.89$ (m, 2H, H-3'), 7.04 (t, $J=8.0 \mathrm{~Hz}, 1 \mathrm{H}$, $\mathrm{H}-5), 7.04-7.10\left(\mathrm{~m}, 2 \mathrm{H}, \mathrm{H}-2^{\prime}\right) .{ }^{13} \mathrm{C}-\mathrm{NMR}\left(75.4 \mathrm{MHz}, \mathrm{CDCl}_{3}\right): \delta=55.5\left(\mathrm{CH}_{3} \mathrm{O}\right), 101.9(\mathrm{C}-2), 106.3$ (C-4), 108.0 (C-6), 114.6 (C-3'), 122.9 (C-2'), 130.3 (C-5), 135.1 (C-1'), 146.9 (C-1), 155.4 (C-4'), 156.7 (C-3). MS (70 eV): m/z (\%) 215 (M+1 100), 201 (6), 185 (7), 172 (5), 146 (4), 132 (5), 91 (11). HRMS (EI): $m / z\left[\mathrm{M}^{+}\right]$calcd for $\mathrm{C}_{13} \mathrm{H}_{13} \mathrm{NO}_{2}: 215.0946$; found: 215.0952 . 
3-(3-Methoxyphenylamino)phenol (6d) [66]. Following the procedure described for 6a, with $4 \mathbf{d d}(0.200$ g, $0.92 \mathrm{mmol})$ and $\mathrm{Pd} / \mathrm{C}(10 \%)(0.060 \mathrm{~g}, 0.057 \mathrm{mmol}), 6 \mathrm{~d}(0.166 \mathrm{~g}, 84 \%)$ was obtained as a purple oil. $R_{\mathrm{f}} 0.51$ (hexane/EtOAc, 8:2). IR (film): $v_{\max } 3411,1645,1489,1156,764 \mathrm{~cm}^{-1} .{ }^{1} \mathrm{H}-\mathrm{NMR}(300 \mathrm{MHz}$, $\left.\mathrm{CDCl}_{3}\right): \delta=3.71\left(\mathrm{~s}, 3 \mathrm{H}, \mathrm{CH}_{3} \mathrm{O}\right), 5.76($ br s, $1 \mathrm{H}, \mathrm{NH}), 6.38$ (ddd, $\left.J=8.1,2.4,0.9 \mathrm{~Hz}, 1 \mathrm{H}, \mathrm{H}-4\right), 6.46$ (dm, $\left.J=7.8 \mathrm{~Hz}, 1 \mathrm{H}, \mathrm{H}-4^{\prime}\right), 6.54$ (t, $\left.J=2.4 \mathrm{~Hz}, 1 \mathrm{H}, \mathrm{H}-2\right), 6.56-6.65$ (m, 3H, H-2', H-6, H-6'), 7.05 (t, $J=8.1 \mathrm{~Hz}, 1 \mathrm{H}, \mathrm{H}-5), 7.11\left(\mathrm{t}, J=7.8 \mathrm{~Hz}, 1 \mathrm{H}, \mathrm{H}-5^{\prime}\right) .{ }^{13} \mathrm{C}-\mathrm{NMR}\left(75.4 \mathrm{MHz}, \mathrm{CDCl}_{3}\right): \delta=55.1\left(\mathrm{CH}_{3} \mathrm{O}\right)$, 103.8 (C-2'), 104.6 (C-2), 106.3 (C-4'), 108.0 (C-4), 110.1 (C-6'), 110.7 (C-6), 130.0 (C-5'), 130.2 (C-5), 144.0 (C-1'), 144.3 (C-1), 156.6 (C-3), 160.3 (C-3'). MS (70 eV): $m / z(\%) 215\left(\mathrm{M}^{+}, 26\right), 199$

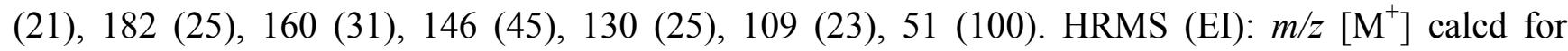
$\mathrm{C}_{13} \mathrm{H}_{13} \mathrm{NO}_{2}$ : 215.0946; found: 215.0952 .

3-(3,5-Dimethoxyphenylamino)phenol (6e). Following the procedure described for $\mathbf{6 a}$, with $4 \mathbf{e}(0.200 \mathrm{~g}$, $0.81 \mathrm{mmol})$ and $\mathrm{Pd} / \mathrm{C}(10 \%)(0.060 \mathrm{~g}, 0.057 \mathrm{mmol}), 6 \mathrm{e}(0.174 \mathrm{~g}, 88 \%)$ was obtained as a yellow oil. $R_{\mathrm{f}} 0.49$ (hexane/EtOAc, 8:2). IR (film): $v_{\max } 3379,2917,1594,1481,1203,1152,1065,821 \mathrm{~cm}^{-1}$. ${ }^{1} \mathrm{H}-\mathrm{NMR}\left(300 \mathrm{MHz}, \mathrm{CDCl}_{3}\right): \delta=3.73\left(\mathrm{~s}, 6 \mathrm{H}, 2 \mathrm{CH}_{3} \mathrm{O}\right), 5.74($ br s, $1 \mathrm{H}, \mathrm{NH}), 6.07(\mathrm{t}, J=2.1 \mathrm{~Hz}, 1 \mathrm{H}$, H-4'), 6.23 (d, $\left.J=2.1 \mathrm{~Hz}, 2 \mathrm{H}, \mathrm{H}-2^{\prime}, \mathrm{H}-6^{\prime}\right), 6.39$ (dd, $\left.J=8.1,2.4 \mathrm{~Hz}, 1 \mathrm{H}, \mathrm{H}-4\right), 6.55$ (dd, $J=2.4,2.1$ $\mathrm{Hz}, 1 \mathrm{H}, \mathrm{H}-2), 6.62$ (ddd, $J=8.1,2.1,0.9 \mathrm{~Hz}, 1 \mathrm{H}, \mathrm{H}-6), 7.08$ (t, $J=8.1 \mathrm{~Hz}, 1 \mathrm{H}, \mathrm{H}-5) .{ }^{13} \mathrm{C}-\mathrm{NMR}(75.4$ $\left.\mathrm{MHz}, \mathrm{CDCl}_{3}\right): \delta=55.3\left(2 \mathrm{CH}_{3} \mathrm{O}\right), 93.2\left(\mathrm{C}^{-} 4^{\prime}\right), 96.3\left(\mathrm{C}-2^{\prime}, \mathrm{C}^{\prime} 6^{\prime}\right), 105.0(\mathrm{C}-2), 108.2(\mathrm{C}-4), 110.6$ (C-6), 130.3 (C-5), 144.1 (C-1'), 144.7 (C-1), 156.6 (C-3), 161.4 (C-3', C-5'). MS (70 eV): m/z (\%) 245 $\left(\mathrm{M}^{+}, 3\right), 154$ (14), 153 (96), 125 (15), 124 (100), 94 (25), 92 (22). HRMS (EI): $m / z$ [M $]$ calcd for $\mathrm{C}_{14} \mathrm{H}_{15} \mathrm{NO}_{3}$ : 245.1052; found: 245.1059 .

3-Methoxy- $N$-(p-tolyl)aniline (5a) [67]. In a threaded ACE glass pressure tube with a sealed Teflon screw cap, under $\mathrm{N}_{2}$ atmosphere, a mixture of $4 \mathbf{a}(0.20 \mathrm{~g}, 1.0 \mathrm{mmol})$ and $\mathrm{Pd} / \mathrm{C}(10 \%)(0.060 \mathrm{~g}$, $0.057 \mathrm{mmol})$ in dry $\mathrm{MeOH}(2.5 \mathrm{~mL})$ was stirred at $210{ }^{\circ} \mathrm{C}$ for $24 \mathrm{~h}$. After removing the solvent under vacuum, $\mathrm{K}_{2} \mathrm{CO}_{3}$, $(0.200 \mathrm{~g}, 1.45 \mathrm{mmol})$ and $\mathrm{CH}_{3} \mathrm{I}(0.281 \mathrm{~g}, 1.98 \mathrm{mmol})$ in dry acetone $(20 \mathrm{~mL})$ were added, and the mixture was heated to reflux for $12 \mathrm{~h}$. The solvent was removed under vacuum and the residue purified by column chromatography over silica gel (20 g/g of crude, hexane/EtOAc, 90:10), to give 5a $(0.176 \mathrm{~g}, 83 \%)$ as a white solid. $R_{\mathrm{f}} 0.60$ (hexane/EtOAc, 7:3); mp 49-50 ${ }^{\circ} \mathrm{C}$. IR (KBr): $v_{\max }$ $3367,1598,1493,1462,1256,1157,1032,950,832,774 \mathrm{~cm}^{-1} .{ }^{1} \mathrm{H}-\mathrm{NMR}\left(500 \mathrm{MHz}, \mathrm{CDCl}_{3}\right): \delta=2.30$ (s, 3H, $\left.\mathrm{CH}_{3}\right), 3.75$ (s, 3H, CH $\left.\mathrm{CH}_{3} \mathrm{O}\right) 6.00$ (br s, $\left.1 \mathrm{H}, \mathrm{NH}\right), 6.42$ (ddd, $\left.J=8.5,2.5,0.5 \mathrm{~Hz}, 1 \mathrm{H}, \mathrm{H}-4\right)$, 6.55-6.59 (m, 2H, H-2, H-6), 6.98-7.02 (m, 2H, H-2'), 7.06-7.10 (m, 2H, H-3'), 7.12 (tm, J=8.5 Hz, 1H, H-5). ${ }^{13} \mathrm{C}-\mathrm{NMR}\left(125 \mathrm{MHz}, \mathrm{CDCl}_{3}\right): \delta=20.7\left(\mathrm{CH}_{3}\right), 55.1\left(\mathrm{CH}_{3} \mathrm{O}\right), 102.4(\mathrm{C}-2), 105.5(\mathrm{C}-4), 109.4$ (C-6), 119.4 (C-2'), 129.8 (C-3'), 130.0 (C-5), 131.2 (C-4'), 140.0 (C-1'), 145.4 (C-1), 160.7 (C-3). MS (70 eV): $m / z(\%) 213\left(\mathrm{M}^{+}, 100\right), 200$ (23), 189 (24), 174 (21), 160 (39), 130 (11), 91 (12), 84 (9). HRMS (EI): $m / z\left[\mathrm{M}^{+}\right]$calcd for $\mathrm{C}_{14} \mathrm{H}_{15} \mathrm{NO}: 213.1154$; found: 213.1153.

3-Methoxy-N-(4-methoxyphenyl)aniline (5b) [29]. Following the procedure described for 5a using $\mathbf{4 b}$ (0.200 g, $0.92 \mathrm{mmol}), \mathrm{Pd} / \mathrm{C}(10 \%)(0.055 \mathrm{~g}, 0.052 \mathrm{mmol}), \mathrm{K}_{2} \mathrm{CO}_{3}(0.190 \mathrm{~g}, 1.38 \mathrm{mmol})$ and MeI (0.261 g, $1.84 \mathrm{mmol}), \mathbf{5 b}(0.179 \mathrm{~g}, 85 \%)$ was obtained as a white solid. $R_{\mathrm{f}} 0.55$ (hexane/EtOAc, 7:3); $\mathrm{mp}$ 99-100 ${ }^{\circ} \mathrm{C}$. IR (KBr): $v_{\max } 3400,1597,1509,1460,1239,1157,1035,825,768 \mathrm{~cm}^{-1} .{ }^{1} \mathrm{H}-\mathrm{NMR}$ $\left(300 \mathrm{MHz}, \mathrm{CDCl}_{3}\right): \delta=3.75\left(\mathrm{~s}, 3 \mathrm{H}, \mathrm{CH}_{3} \mathrm{O}\right), 3.79$ (s, 3H, $\left.\mathrm{CH}_{3} \mathrm{O}\right), 6.51$ (br s, $\left.1 \mathrm{H}, \mathrm{NH}\right), 6.38$ (br dd, 
$J=7.8,2.1 \mathrm{~Hz}, 1 \mathrm{H}, \mathrm{H}-4), 6.44-6.51$ (m, 2H, H-2, H-6), 6.83-6.89 (m, 2H, H-3'), 7.04-7.10 (m, 2H, $\left.\mathrm{H}-2^{\prime}\right), 7.11(\mathrm{t}, J=7.8 \mathrm{~Hz}, 1 \mathrm{H}, \mathrm{H}-5) .{ }^{13} \mathrm{C}-\mathrm{NMR}\left(75.4 \mathrm{MHz}, \mathrm{CDCl}_{3}\right): \delta=55.1\left(\mathrm{CH}_{3} \mathrm{O}-\mathrm{C} 3\right), 55.5$ $\left(\mathrm{CH}_{3} \mathrm{O}-\mathrm{C} 4^{\prime}\right), 101.1$ (C-2), 104.6 (C-4), 108.2 (C-6), 114.6 (C-3'), 122.7 (C-2'), 130.0 (C-5), 135.3 (C-1'), 146.6 (C-1), 155.4 (C-4'), 160.7 (C-3). MS (70 eV): m/z (\%) 229 (M+, 90), 216 (47), 214 (100), 186 (19), 171 (21), 142 (15), 115 (21). HRMS (EI): $m / z$ [M $]$ calcd for $\mathrm{C}_{14} \mathrm{H}_{15} \mathrm{NO}_{2}$ : 229.1103; found: 229.1111.

3-Methoxy- $N$-(m-tolyl)aniline (5c). Following the procedure described for 5a, with $4 \mathbf{c}(0.20 \mathrm{~g}$, $1.0 \mathrm{mmol}), \mathrm{Pd} / \mathrm{C}(10 \%)(0.060 \mathrm{~g}, 0.057 \mathrm{mmol}), \mathrm{K}_{2} \mathrm{CO}_{3}(0.200 \mathrm{~g}, 1.45 \mathrm{mmol})$ and $\mathrm{MeI}(0.281 \mathrm{~g}$, $1.98 \mathrm{mmol}), \mathbf{5 c}(0.18 \mathrm{~g}, 87 \%)$ was obtained as a yellow oil. $R_{\mathrm{f}} 0.59$ (hexane/EtOAc, 7:3). IR (film): $v_{\max } 3391,1589,1490,1266,1203,1155,1042,765,687 \mathrm{~cm}^{-1} .{ }^{1} \mathrm{H}-\mathrm{NMR}\left(300 \mathrm{MHz}, \mathrm{CDCl}_{3}\right): \delta=2.31$ (s, 3H, $\left.\mathrm{CH}_{3}\right), 3.77$ (s, 3H, $\mathrm{CH}_{3} \mathrm{O}$ ), 5.67 (br s, 1H, NH), 6.47 (br dd, $\left.J=7.8,2.4 \mathrm{~Hz}, 1 \mathrm{H}, \mathrm{H}-4\right), 6.62-6.67$ (m, 2H, H-2, H-6), 6.76 (br d, $J=7.2$ Hz, 1H, H-4'), 6.88-6.94 (m, 2H, H-2', H-6'), 7.12-7.20 (m, 2H, $\left.\mathrm{H}-5, \mathrm{H}-5^{\prime}\right) .{ }^{13} \mathrm{C}-\mathrm{NMR}\left(75.4 \mathrm{MHz}, \mathrm{CDCl}_{3}\right): \delta=21.5\left(\mathrm{CH}_{3}\right), 55.2\left(\mathrm{CH}_{3} \mathrm{O}\right), 103.2(\mathrm{C}-2), 105.9(\mathrm{C}-4)$, 110.2 (C-6), 115.4 (C-6'), 119.0 (C-2'), 122.1 (C-4'), 129.1 (C-5'), 130.0 (C-5), 139.2 (C-3'), 142.7 (C-1'), 144.6 (C-1), 160.6 (C-3). MS (70 eV): m/z (\%) 213 (M+100), 200 (32), 189 (35), 174 (26), 160 (44), 130 (13), 92 (11), 77 (11). HRMS (EI): $m / z$ [M $]$ calcd for $\mathrm{C}_{14} \mathrm{H}_{15} \mathrm{NO}$ : 213.1154; found: 213.1161.

bis(3-Methoxyphenyl)amine (5d) [26]. Following the procedure described for 5a, with $4 \mathbf{d}(0.20 \mathrm{~g}$, $0.92 \mathrm{mmol}), \mathrm{Pd} / \mathrm{C}(10 \%)(0.055 \mathrm{~g}, 0.052 \mathrm{mmol}), \mathrm{K}_{2} \mathrm{CO}_{3}(0.190 \mathrm{~g}, 1.38 \mathrm{mmol})$ and $\mathrm{MeI}(0.261 \mathrm{~g}$, $1.84 \mathrm{mmol})$, 5d (0.171 g, 81\%) was obtained as a white solid. $R_{\mathrm{f}} 0.55$ (hexane/EtOAc, 7:3); mp 154-155 ${ }^{\circ} \mathrm{C}$. IR (film): $v_{\max } 3393,1592,1490,1270,1207,1155,1040,832,760,685 \mathrm{~cm}^{-1} .{ }^{1} \mathrm{H}-\mathrm{NMR}$ $\left(300 \mathrm{MHz}, \mathrm{CDCl}_{3}\right): \delta=3.74\left(\mathrm{~s}, 6 \mathrm{H}, 2 \mathrm{CH}_{3} \mathrm{O}\right), 5.78($ br s, $1 \mathrm{H}, \mathrm{NH}), 6.47(\mathrm{ddm}, J=8.1,2.4 \mathrm{~Hz}, 2 \mathrm{H}, \mathrm{H}-4$, H-4'), 6.61-6.68 (m, 4H, H-2, H-2', H-6, H-6'), 7.14 (t, $\left.J=8.1 \mathrm{~Hz}, 2 \mathrm{H}, \mathrm{H}-5, \mathrm{H}-5^{\prime}\right) .{ }^{13} \mathrm{C}-\mathrm{NMR}(75.4$ $\left.\mathrm{MHz}, \mathrm{CDCl}_{3}\right): \delta=55.1\left(2 \mathrm{CH}_{3} \mathrm{O}\right), 103.6\left(\mathrm{C}-2, \mathrm{C}-2^{\prime}\right), 106.3\left(\mathrm{C}-4, \mathrm{C}-4^{\prime}\right), 110.4\left(\mathrm{C}-6, \mathrm{C}-6{ }^{\prime}\right), 130.0(\mathrm{C}-5$, C-5'), 144.1 (C-1, C-1'), 160.5 (C-3, C-3'). MS (70 eV): m/z (\%) 229 (M+1, 100), 217 (10), 200 (12), 189 (6), 170 (11), 160 (9), 154 (12), 142 (9), 115 (5). HRMS (EI): $m / z$ [M $]$ calcd for $\mathrm{C}_{14} \mathrm{H}_{15} \mathrm{NO}_{2}$ : 229.1103; found: 229.1104 .

3,5-Dimethoxy-N-(3-methoxyphenyl)aniline (5e). Following the procedure described for 5a, with $4 \mathbf{e}$ (0.200 g, $0.818 \mathrm{mmol}), \mathrm{Pd} / \mathrm{C}(10 \%)(0.050 \mathrm{~g}, 0.047 \mathrm{mmol}), \mathrm{K}_{2} \mathrm{CO}_{3}(0.167 \mathrm{~g}, 1.21 \mathrm{mmol})$ and MeI $(0.230 \mathrm{~g}, 1.62 \mathrm{mmol})$, 5e $(0.18 \mathrm{~g}, 86 \%)$ was obtained as a colorless oil. $R_{\mathrm{f}} 0.52$ (hexane/EtOAc, $\left.7: 3\right)$. IR (film): $v_{\max } 3735,1590,1541,1457,1203,1150,1057 \mathrm{~cm}^{-1} .{ }^{1} \mathrm{H}-\mathrm{NMR}\left(500 \mathrm{MHz}, \mathrm{CDCl}_{3}\right): \delta=3.74$ (s, $\left.6 \mathrm{H}, 2 \mathrm{CH}_{3} \mathrm{O}\right), 3.76$ (s, 3H, $\left.\mathrm{CH}_{3} \mathrm{O}-3\right), 5.73$ (br s, $\left.1 \mathrm{H}, \mathrm{NH}\right), 6.07$ (t, $\left.J=2.0 \mathrm{~Hz}, 1 \mathrm{H}, \mathrm{H}-4\right), 6.24$ (d, $J=2.0 \mathrm{~Hz}, 2 \mathrm{H}, \mathrm{H}-2, \mathrm{H}-6), 6.49$ (ddd, $\left.J=8.0,2.0,1.0 \mathrm{~Hz}, 1 \mathrm{H}, \mathrm{H}-4^{\prime}\right), 6.65$ (t, $\left.J=2.0 \mathrm{~Hz}, 1 \mathrm{H}, \mathrm{H}-2^{\prime}\right)$, 6.67 (ddd, $\left.J=8.0,2.0,1.0 \mathrm{~Hz}, 1 \mathrm{H}, \mathrm{H}-6^{\prime}\right), 7.15\left(\mathrm{t}, J=8.0 \mathrm{~Hz}, 1 \mathrm{H}, \mathrm{H}-5^{\prime}\right) .{ }^{13} \mathrm{C}-\mathrm{NMR}\left(125 \mathrm{MHz}, \mathrm{CDCl}_{3}\right)$ : $\delta=55.1\left(\mathrm{CH}_{3} \mathrm{O}-3^{\prime}\right), 55.2\left(2 \mathrm{CH}_{3} \mathrm{O}\right), 93.3(\mathrm{C}-4), 96.2(\mathrm{C}-2, \mathrm{C}-6), 104.2\left(\mathrm{C}-2^{\prime}\right), 106.7\left(\mathrm{C}-4^{\prime}\right), 111.0$ (C-6'), $130.0\left(\mathrm{C}-5^{\prime}\right), 144.0$ (C-1), 144.9 (C-1'), 160.6 (C-3'), 161.6 (C-3, C-5). MS (70 eV): m/z (\%) 259 (M+ 7$), 257$ (97), 242 (100), 214 (49), 199 (42), 184 (13), 156 (8), 128 (7). HRMS (EI): $m / z$ [M $\left.{ }^{+}\right]$ calcd for $\mathrm{C}_{15} \mathrm{H}_{17} \mathrm{NO}_{3}$ : 259.1208; found: 259.1209 .

2-Methoxy-6-methyl-9H-carbazole (1h). A mixture of 5a (0.100 g, $0.47 \mathrm{mmol}), \mathrm{Pd}(\mathrm{AcO})_{2}(0.0105 \mathrm{~g}$, $0.047 \mathrm{mmol})$ and $\mathrm{Cu}(\mathrm{AcO})_{2}(0.211 \mathrm{~g}, 1.17 \mathrm{mmol})$ in dry DMF $(0.5 \mathrm{~mL})$, under $\mathrm{N}_{2}$ atmosphere, was stirred and heated at $130{ }^{\circ} \mathrm{C}$ for $70 \mathrm{~min}$ under MW irradiation $(100 \mathrm{~W})$. The solvent was removed 
under vacuum by adding toluene, and the azeotropic distillation was continued until no solvent remained. The residue was purified by column chromatography over silica gel $(10 \mathrm{~g} / \mathrm{g}$ of crude, hexane/EtOAc, 95:5), to give $\mathbf{1 h}(0.079 \mathrm{~g}, 80 \%)$ as a white solid. $R_{\mathrm{f}} 0.60$ (hexane/EtOAc, 7:3); mp 226-227 ${ }^{\circ} \mathrm{C}$ [Lit. [32] 227-228 ${ }^{\circ} \mathrm{C}$ ]. IR (film): $v_{\max } 3392,1659,1026,826,764,687 \mathrm{~cm}^{-1} .{ }^{1} \mathrm{H}-\mathrm{NMR}$ $\left(500 \mathrm{MHz}, \mathrm{DMSO}-d_{6} /\right.$ acetone- $\left.d_{6}, 3: 7\right): \delta=2.46\left(\mathrm{~s}, 3 \mathrm{H}, \mathrm{CH}_{3}\right), 3.86\left(\mathrm{~s}, 3 \mathrm{H}, \mathrm{CH}_{3} \mathrm{O}\right), 6.76(\mathrm{dd}, J=8.4$, $2.4 \mathrm{~Hz}, 1 \mathrm{H}, \mathrm{H}-3), 6.99$ (d, $J=2.4 \mathrm{~Hz}, 1 \mathrm{H}, \mathrm{H}-1), 7.11$ (dd, $J=8.2,1.5 \mathrm{~Hz}, 1 \mathrm{H}, \mathrm{H}-7), 7.33$ (d, $J=8.2 \mathrm{~Hz}, 1 \mathrm{H}, \mathrm{H}-8), 7.78$ (br s, $1 \mathrm{H}, \mathrm{H}-5), 7.91$ (d, $J=8.4 \mathrm{~Hz}, 1 \mathrm{H}, \mathrm{H}-4), 10.6$ (br s, 1H, NH). ${ }^{13} \mathrm{C}-\mathrm{NMR}\left(125 \mathrm{MHz}, \mathrm{DMSO}-d_{6} /\right.$ acetone- $\left.d_{6}, 3: 7\right): \delta=21.2\left(\mathrm{CH}_{3}\right), 55.4\left(\mathrm{CH}_{3} \mathrm{O}\right), 95.0(\mathrm{C}-1), 108.1$ (C-3), 110.8 (C-8), 117.2 (C-4a), 119.6 (C-5), 121.1 (C-4), 124.0 (C-4a), 125.9 (C-7), 128.0 (C-6), 139.0 (C-8a), 142.4 (C-9a), 159.5 (C-2). MS (70 eV): m/z (\%) $211\left(\mathrm{M}^{+}, 100\right), 196$ (51), 168 (76), 139 (10), 86 (6). HRMS (EI): $m / z\left[\mathrm{M}^{+}\right]$calcd for $\mathrm{C}_{14} \mathrm{H}_{13} \mathrm{NO}: 211.0997$; found: 211.0994.

2,6-Dimethoxy-9H-carbazole (1i). Following the procedure described for $\mathbf{1 h}$, with $\mathbf{5 b}(0.100 \mathrm{~g}, 0.44$ $\mathrm{mmol}), \mathrm{Pd}(\mathrm{AcO})_{2}(0.0099 \mathrm{~g}, 0.044 \mathrm{mmol})$ and $\mathrm{Cu}(\mathrm{AcO})_{2}(0.198 \mathrm{~g}, 1.1 \mathrm{mmol}), \mathbf{1 i}(0.086 \mathrm{~g}, 87 \%)$ was obtained as a white solid. $R_{\mathrm{f}} 0.55$ (hexane/EtOAc, 7:3); mp $162-163{ }^{\circ} \mathrm{C}$ [Lit. [29] $163-164{ }^{\circ} \mathrm{C}$ ]. IR $(\mathrm{KBr}): v_{\max } 3398,1626,1491,1465,1284,1221,1202,1162,1029,820 \mathrm{~cm}^{-1}$. ${ }^{1} \mathrm{H}-\mathrm{NMR}(300 \mathrm{MHz}$, $\left.\mathrm{CDCl}_{3}\right): \delta=3.85\left(\mathrm{~s}, 3 \mathrm{H}, \mathrm{CH}_{3} \mathrm{O}\right), 3.86\left(\mathrm{~s}, 3 \mathrm{H}, \mathrm{CH}_{3} \mathrm{O}\right), 6.75(\mathrm{dd}, J=8.4,2.4 \mathrm{~Hz}, 1 \mathrm{H}, \mathrm{H}-3), 6.92(\mathrm{dd}$, $J=8.7,2.5 \mathrm{~Hz}, 1 \mathrm{H}, \mathrm{H}-7), 6.95(\mathrm{~d}, J=2.4 \mathrm{~Hz}, 1 \mathrm{H}, \mathrm{H}-1), 7.31(\mathrm{~d}, J=8.7 \mathrm{~Hz}, 1 \mathrm{H}, \mathrm{H}-8), 7.51(\mathrm{~d}$, $J=2.5 \mathrm{~Hz}, 1 \mathrm{H}, \mathrm{H}-5), 7.89$ (d, $J=8.4 \mathrm{~Hz}, 1 \mathrm{H}, \mathrm{H}-4), 9.90($ br s, $1 \mathrm{H}, \mathrm{NH}) .{ }^{13} \mathrm{C}-\mathrm{NMR}(75.4 \mathrm{MHz}$, $\left.\mathrm{CDCl}_{3}\right): \delta=53.9\left(\mathrm{CH}_{3} \mathrm{O}\right), 54.3\left(\mathrm{CH}_{3} \mathrm{O}\right), 93.3(\mathrm{C}-1), 101.2(\mathrm{C}-5), 106.6(\mathrm{C}-3), 110.0(\mathrm{C}-8), 112.0$ (C-7), 115.9 (C-4a), 119.7 (C-4), 122.7 (C-4b), 133.8 (C-8a), 141.1 (C-9a), 152.8 (C-6), 158.0 (C-2). MS (70 eV): $m / z(\%) 227\left(\mathrm{M}^{+}, 100\right), 212$ (86), 184 (69), 169 (28), 141 (19), 114 (7). HRMS (EI): $m / z$ $\left[\mathrm{M}^{+}\right]$calcd for $\mathrm{C}_{14} \mathrm{H}_{13} \mathrm{NO}_{2}$ : 227.0946; found: 227.0951 .

2-Methoxy-7-methyl-9H-carbazole (1j). Following the procedure described for $\mathbf{1 h}$, with $\mathbf{5 c}(0.100 \mathrm{~g}$, $0.47 \mathrm{mmol}), \mathrm{Pd}(\mathrm{AcO})_{2}(0.0105 \mathrm{~g}, 0.047 \mathrm{mmol})$ and $\mathrm{Cu}(\mathrm{AcO})_{2}(0.211 \mathrm{~g}, 1.17 \mathrm{mmol}), \mathbf{1 j}(0.081 \mathrm{~g}, 82 \%)$ was obtained as a white solid. $R_{\mathrm{f}} 0.61$ (hexane/EtOAc, 7:3); mp $162-163{ }^{\circ} \mathrm{C}$ [Lit. [68] $280{ }^{\circ} \mathrm{C}$ ]. IR (film): $v_{\max } 3399,1654,1047,1025,995,827,766 \mathrm{~cm}^{-1} .{ }^{1} \mathrm{H}-\mathrm{NMR}$ (500 MHz, DMSO- $d_{6} /$ acetone- $d_{6}$, 3:7): $\delta=2.46\left(\mathrm{~s}, 3 \mathrm{H}, \mathrm{CH}_{3}\right), 3.85\left(\mathrm{~s}, 3 \mathrm{H}, \mathrm{CH}_{3} \mathrm{O}\right), 6.75(\mathrm{dd}, J=8.5,2.5 \mathrm{~Hz}, 1 \mathrm{H}, \mathrm{H}-3), 6.95(\mathrm{dd}, J=8.3$, $1.0 \mathrm{~Hz}, 1 \mathrm{H}, \mathrm{H}-6), 6.99$ (d, $J=2.5 \mathrm{~Hz}, 1 \mathrm{H}, \mathrm{H}-1$ ), 7.25 (br s, 1H, H-8), 7.84 (d, J=8.3 Hz, 1H, H-5), 7.88 (d, $J=8.5 \mathrm{~Hz}, 1 \mathrm{H}, \mathrm{H}-4), 10.7$ (br s, $1 \mathrm{H}, \mathrm{NH}) .{ }^{13} \mathrm{C}-\mathrm{NMR}\left(125 \mathrm{MHz}\right.$, DMSO- $d_{6} /$ acetone- $\left.d_{6}, 3: 7\right)$ : $\delta=21.4\left(\mathrm{CH}_{3}\right), 54.9\left(\mathrm{CH}_{3} \mathrm{O}\right), 94.2(\mathrm{C}-1), 106.9(\mathrm{C}-3), 110.3(\mathrm{C}-8), 116.1(\mathrm{C}-4 \mathrm{a}), 118.4(\mathrm{C}-5), 119.6$ (C-6), 119.9 (C-4), 120.2 (C-4b), 133.1 (C-7), 140.0 (C-8a), 140.8 (C-9a), 157.8 (C-2). MS (70 eV):

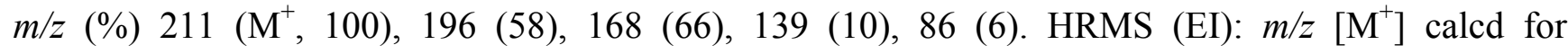
$\mathrm{C}_{14} \mathrm{H}_{13} \mathrm{NO}: 211.0997$; found: 211.1000 .

2,7-Dimethoxy-9H-carbazole (Clausine V, 1d). Following the procedure described for $\mathbf{1 h}$, with $\mathbf{5 d}$ (0.100 g, $0.44 \mathrm{mmol}), \mathrm{Pd}(\mathrm{AcO})_{2}(0.0099 \mathrm{~g}, 0.044 \mathrm{mmol})$ and $\mathrm{Cu}(\mathrm{AcO})_{2}(0.198 \mathrm{~g}, 1.10 \mathrm{mmol}), \mathbf{1 d}$ $(0.089 \mathrm{~g}, 90 \%)$ was obtained as a white solid. $R_{\mathrm{f}} 0.56$ (hexane/EtOAc, 7:3); mp 229-230 ${ }^{\circ} \mathrm{C}$ [Lit. [22] 228-230 $\left.{ }^{\circ} \mathrm{C}\right]$. IR (KBr): $v_{\max } 3382,2927,1608,1575,1502,1457,1322,1265,1233,1160,1118,1026$, $825,805 \mathrm{~cm}^{-1} .{ }^{1} \mathrm{H}-\mathrm{NMR}\left(300 \mathrm{MHz}, \mathrm{DMSO}-d_{6} /\right.$ acetone- $\left.d_{6}, 3: 7\right): \delta=3.85\left(\mathrm{~s}, 6 \mathrm{H}, 2 \mathrm{CH}_{3} \mathrm{O}\right), 6.75(\mathrm{dd}$, $J=8.4,2.4$ Hz, 2H, H-3, H-6), 6.99 (d, $J=2.4$ Hz, 2H, H-1, H-8), 7.85 (d, $J=8.4$ Hz, 2H, H-4, H-5), 
10.81 (br s, $1 \mathrm{H}, \mathrm{NH}) .{ }^{13} \mathrm{C}-\mathrm{NMR}\left(75.4 \mathrm{MHz}, \mathrm{DMSO}-d_{6} /\right.$ acetone- $\left.d_{6}, 3: 7\right): \delta=54.9\left(2 \mathrm{CH}_{3} \mathrm{O}\right), 94.6(\mathrm{C}-1$, C8), 107.3 (C-3, C-6), 116.8 (C-4a, C-4b), 119.7 (C-4, C-5), 141.4 (C-8a, C-9a), 157.9 (C-2, C-7). MS (70 eV): $m / z(\%) 227\left(\mathrm{M}^{+}, 77\right), 212$ (100), 184 (42), 169 (54), 153 (13), 141 (27), 114 (5). HRMS (EI): $m / z\left[\mathrm{M}^{+}\right]$calcd for $\mathrm{C}_{14} \mathrm{H}_{13} \mathrm{NO}_{2}$ : 227.0946; found: 227.0946 .

2,4,7-Trimethoxy-9H-carbazole (1k). Following the procedure described for $\mathbf{1 h}$, with $\mathbf{5 e}(0.101 \mathrm{~g}, 0.39$ $\mathrm{mmol}), \mathrm{Pd}(\mathrm{AcO})_{2}(0.0087 \mathrm{~g}, 0.039 \mathrm{mmol})$ and $\mathrm{Cu}(\mathrm{AcO})_{2}(0.175 \mathrm{~g}, 0.97 \mathrm{mmol}), \mathbf{1 k}(0.092 \mathrm{~g}, 92 \%)$ was obtained as a white solid. $R_{\mathrm{f}} 0.20$ (hexane/EtOAc, 7:3); $\mathrm{mp} 167-168{ }^{\circ} \mathrm{C}$. IR (KBr): $v_{\max } 3383,1617$, 1580, 1510, 1453, 1428, 1260, 1213, 1149, 1119, 1032, $803 \mathrm{~cm}^{-1} .{ }^{1} \mathrm{H}-\mathrm{NMR}(500 \mathrm{MHz}$, $\mathrm{CDCl}_{3}$ /acetone- $\left.d_{6}, 7: 3\right): \delta=3.83\left(\mathrm{~s}, 6 \mathrm{H}, 2 \mathrm{CH}_{3} \mathrm{O}\right), 3.98\left(\mathrm{~s}, 3 \mathrm{H}, \mathrm{CH}_{3} \mathrm{O}\right), 6.27(\mathrm{~d}, J=1.3 \mathrm{~Hz}, 1 \mathrm{H}, \mathrm{H}-3)$, $6.46(\mathrm{~d}, J=1.3 \mathrm{~Hz}, 1 \mathrm{H}, \mathrm{H}-1), 6.76$ (dd, $J=8.5,2.2 \mathrm{~Hz}, 1 \mathrm{H}, \mathrm{H}-6), 6.82$ (d, $J=2.2 \mathrm{~Hz}, 1 \mathrm{H}, \mathrm{H}-8), 7.99$ $(\mathrm{d}, J=8.5 \mathrm{~Hz}, 1 \mathrm{H}, \mathrm{H}-5), 9.32$ (br s, $1 \mathrm{H}, \mathrm{NH}) .{ }^{13} \mathrm{C}-\mathrm{NMR}\left(125 \mathrm{MHz}, \mathrm{CDCl}_{3} /\right.$ acetone- $\left._{6}, 7: 3\right): \delta=54.7$ $\left(\mathrm{CH}_{3} \mathrm{O}\right), 54.9\left(\mathrm{CH}_{3} \mathrm{O}\right), 55.0\left(\mathrm{CH}_{3} \mathrm{O}\right), 86.7(\mathrm{C}-1), 90.2(\mathrm{C}-3), 94.0(\mathrm{C}-8), 106.0(\mathrm{C}-4 \mathrm{a}), 107.0(\mathrm{C}-6)$, 116.1 (C-4b), 121.7 (C-5), 139.7 (C-8a), 141.3 (C-9a), 155.2 (C-4), 156.8 (C-7), 158.7 (C-2). MS (70 eV): $m / z(\%) 257\left(\mathrm{M}^{+}, 39\right), 247$ (36), 230 (100), 219 (24), 214 (22), 190 (82), 176 (21), 160 (19), 117 (7). HRMS (EI): $m / z\left[\mathrm{M}^{+}\right]$calcd for $\mathrm{C}_{15} \mathrm{H}_{15} \mathrm{NO}_{3}$ : 257.1052; found: 257.1052.

7-Methoxy-9H-carbazole-3-carbaldehyde (Clauszoline-K) (1f). A mixture of $\mathbf{1 h}(0.030 \mathrm{~g}, 0.14 \mathrm{mmol})$ and DDQ (0.129 g, $0.57 \mathrm{mmol})$ in acetone $/ \mathrm{MeOH} / \mathrm{H}_{2} \mathrm{O}(1: 1: 1)(10 \mathrm{~mL})$ was stirred at $25^{\circ} \mathrm{C}$ for $45 \mathrm{~min}$. The solvent was removed under vacuum and the residue purified by column chromatography over silica gel (10 g/g of crude, hexane/EtOAc, 8:2), to give $\mathbf{1 f}(0.022 \mathrm{~g}, 70 \%)$ as a white solid. $R_{\mathrm{f}} 0.25$ (hexane/EtOAc, 8:2); mp $184-185^{\circ} \mathrm{C}$ [Lit. [32] $183-186{ }^{\circ} \mathrm{C}$ ]. IR (KBr): $v_{\max } 3296,1670,1604,1570$, 1479, 1322, 1237, 1160, 1026, $821 \mathrm{~cm}^{-1} .{ }^{1} \mathrm{H}-\mathrm{NMR}$ (500 MHz, DMSO- $\left.d_{6} / \mathrm{CDCl}_{3}, 3: 7\right): \delta=3.88(\mathrm{~s}, 3 \mathrm{H}$, $\left.\mathrm{CH}_{3} \mathrm{O}\right), 6.84(\mathrm{dd}, J=8.5,2.0 \mathrm{~Hz}, 1 \mathrm{H}, \mathrm{H}-6), 7.00$ (d, $\left.J=2.0 \mathrm{~Hz}, 1 \mathrm{H}, \mathrm{H}-8\right), 7.51$ (d, $\left.J=8.0,1 \mathrm{H}, \mathrm{H}-1\right)$, $7.83(\mathrm{dd}, J=8.0,1.0 \mathrm{~Hz}, 1 \mathrm{H}, \mathrm{H}-2), 7.96$ (d, $J=8.5 \mathrm{~Hz}, 1 \mathrm{H}, \mathrm{H}-5), 8.45$ (s, 1H, H-4), 10.05 (s, 1H, $\mathrm{CHO}$ ), 11.40 (br s, $1 \mathrm{H}, \mathrm{NH}) .{ }^{13} \mathrm{C}-\mathrm{NMR}\left(125 \mathrm{MHz}, \mathrm{DMSO}-d_{6} / \mathrm{CDCl}_{3}, 3: 7\right): \delta=54.0\left(\mathrm{CH}_{3} \mathrm{O}\right), 93.8$ (C-8), 107.6 (C-6), 109.5 (C-1), 115.0 (C-4b), 119.7 (C-5), 121.3 (C-4), 121.8 (C-4a), 124.1 (C-2), 126.9 (C-3), 140.8 (C-8a), 142.6 (C-9a), 158.0 (C-7), 190.2 (CHO). MS (70 eV): m/z (\%) $225\left(\mathrm{M}^{+}\right.$, 40), 210 (28), 180 (72), 167 (97), 160 (44), 146 (30), 130 (32), 115 (28), 77 (29), 51 (100). HRMS (EI): $m / z\left[\mathrm{M}^{+}\right]$calcd for $\mathrm{C}_{14} \mathrm{H}_{11} \mathrm{NO}_{2}: 225.0790$; found: 225.0796 .

Methyl 7-methoxy-9H-carbazole-3-carboxylate (Clauszoline-L, Clausine C) (1g). A mixture of $\mathbf{1 f}$ (0.200 g, $0.89 \mathrm{mmol}), \mathrm{MnO}_{2}(0.20 \mathrm{~g}, 2.3 \mathrm{mmol})$, and $\mathrm{KCN}(0.028 \mathrm{~g}, 0.43 \mathrm{mmol})$ in $\mathrm{MeOH}(10 \mathrm{~mL})$ was stirred at $25{ }^{\circ} \mathrm{C}$ for $24 \mathrm{~h}$. The solvent was removed under vacuum and the residue purified by column chromatography over silica gel (10 g/g of crude, hexane/EtOAc, 8:2), to give $\mathbf{1 g}(0.22 \mathrm{~g}, 97 \%)$ as a white solid. $R_{\mathrm{f}} 0.29$ (hexane/EtOAc, 8:2); mp 194-195 ${ }^{\circ} \mathrm{C}$ [Lit. [20] 195-197 ${ }^{\circ} \mathrm{C}$; [32] $195{ }^{\circ} \mathrm{C}$; [33] $\left.194-195{ }^{\circ} \mathrm{C}\right]$. IR (KBr): $v_{\max } 3288,1698,1605,1439,1327,1259,1195,1159,1094,815,728 \mathrm{~cm}^{-1}$. ${ }^{1} \mathrm{H}-\mathrm{NMR}\left(500 \mathrm{MHz}\right.$, acetone- $\left.d_{6}\right): \delta=3.88\left(\mathrm{~s}, 3 \mathrm{H}, \mathrm{CH}_{3} \mathrm{O}\right), 3.91\left(\mathrm{~s}, 3 \mathrm{H}, \mathrm{CH}_{3} \mathrm{O}\right), 6.88(\mathrm{dd}, J=8.5,2.0$ $\mathrm{Hz}, 1 \mathrm{H}, \mathrm{H}-6), 7.08$ (d, $J=2.0 \mathrm{~Hz}, 1 \mathrm{H}, \mathrm{H}-8), 7.51$ (d, $J=8.5,1 \mathrm{H}, \mathrm{H}-1), 7.99$ (dd, $J=8.5,1.5 \mathrm{~Hz}, 1 \mathrm{H}$, H-2), 8.09 (d, $J=8.5 \mathrm{~Hz}, 1 \mathrm{H}, \mathrm{H}-5), 8.69$ (d, $J=1.5 \mathrm{~Hz}, 1 \mathrm{H}, \mathrm{H}-4), 10.80$ (br s, $1 \mathrm{H}, \mathrm{NH}) .{ }^{13} \mathrm{C}-\mathrm{NMR}$ $\left(125 \mathrm{MHz}\right.$, acetone- $\left.d_{6}\right): \delta=51.8\left(\mathrm{CH}_{3} \mathrm{O}\right), 55.7\left(\mathrm{CH}_{3} \mathrm{O}\right), 95.7(\mathrm{C}-8), 109.6(\mathrm{C}-6), 110.9(\mathrm{C}-1), 117.4$ (C-4b), 121.6 (C-5), 121.9 (C-4a), 122.1 (C-4), 123.9 (C-3), 126.4 (C-2), 143.0 (C-8a), 144.0 (C-9a), 
160.5 (C-2), $167.9\left(\mathrm{CO}_{2} \mathrm{Me}\right)$. MS (70 eV): m/z (\%) $255\left(\mathrm{M}^{+}, 100\right), 240$ (22), 224 (44), 212 (61), 196 (38), 181 (33), 153 (67), 126 (15), 84 (20), 51 (21). HRMS (EI): $m / z$ [M $]$ calcd for $\mathrm{C}_{15} \mathrm{H}_{13} \mathrm{NO}_{3}$ : 255.0895; found: 255.0900 .

6-Methyl-2,3-dihydro-1H-carbazol-4(9H)-one (7a). In a threaded ACE glass pressure tube with a sealed Teflon screw cap, under $\mathrm{N}_{2}$ atmosphere, a mixture of $4 \mathrm{a}(0.10 \mathrm{~g}, 0.5 \mathrm{mmol})$ and $\mathrm{Pd}(\mathrm{AcO})_{2}$ $(0.034 \mathrm{~g}, 0.15 \mathrm{mmol})$ in dry $\mathrm{MeCN}(2.5 \mathrm{~mL})$ was stirred at $80{ }^{\circ} \mathrm{C}$ for $24 \mathrm{~h}$. The solvent was removed under vacuum and the residue purified by column chromatography over silica gel $(20 \mathrm{~g} / \mathrm{g}$ of crude, hexane/EtOAc, 80:20), to give $7 \mathbf{a}(0.077 \mathrm{~g}, 78 \%)$ as a white solid. $R_{\mathrm{f}} 0.15$ (hexane/EtOAc, $\left.1: 1\right)$; mp 281-282 ${ }^{\circ} \mathrm{C}$ [Lit. [69] 280-282 ${ }^{\circ} \mathrm{C}$ ]. IR (film): $v_{\max } 3154,2934,1615,1469,1406,1375,1213,1183$, 1122, 1070, 1016, $797 \mathrm{~cm}^{-1} .{ }^{1} \mathrm{H}-\mathrm{NMR}\left(300 \mathrm{MHz}, \mathrm{DMSO}-d_{6}\right): \delta=2.09$ (qu, $J=6.3 \mathrm{~Hz}, 2 \mathrm{H}, \mathrm{H}-2$ ), 2.38 (s, 3H, $\left.\mathrm{CH}_{3}\right), 2.41$ (t, $\left.J=6.3 \mathrm{~Hz}, 2 \mathrm{H}, \mathrm{H}-3\right), 2.93$ (t, $\left.J=6.3 \mathrm{~Hz}, 2 \mathrm{H}, \mathrm{H}-1\right), 6.97$ (br d, $J=8.1 \mathrm{~Hz}, 1 \mathrm{H}$, $\mathrm{H}-7), 7.27$ (d, $J=8.1 \mathrm{~Hz}, 1 \mathrm{H}, \mathrm{H}-8), 7.77$ (br s, 1H, H-5), 11.75 (br s, 1H, NH) ${ }^{13} \mathrm{C}-\mathrm{NMR}(75.4 \mathrm{MHz}$, DMSO- $\left.d_{6}\right): \delta=21.2\left(\mathrm{CH}_{3}\right), 22.7(\mathrm{C}-1), 23.4(\mathrm{C}-2), 37.8(\mathrm{C}-3), 111.1(\mathrm{C}-8), 111.4(\mathrm{C}-4 \mathrm{a}), 120.1(\mathrm{C}-5)$, 123.7 (C-7), 124.7 (C-4b), 130.2 (C-6), 134.1 (C-8a), 152.2 (C-9a), 192.8 (C-4). MS (70 eV): m/z (\%) $199\left(\mathrm{M}^{+}, 100\right), 198$ (45), 183 (13), 170 (11), 154 (20), 128 (8), 91 (40).

6-Methoxy-2,3-dihydro-1H-carbazol-4(9H)-one (7b). Following the procedure described for 7a, with 4b $(0.100 \mathrm{~g}, 0.46 \mathrm{mmol})$ and $\mathrm{Pd}(\mathrm{AcO})_{2}(0.0309 \mathrm{~g}, 0.138 \mathrm{mmol}), 7 \mathbf{b}(0.08 \mathrm{~g}, 80 \%)$ was obtained as a white solid. $R_{\mathrm{f}} 0.13$ (hexane/EtOAc, 1:1); mp 252-253 ${ }^{\circ} \mathrm{C}$ [Lit. [70] 250-254 ${ }^{\circ} \mathrm{C}$ ]. IR (KBr): $v_{\max } 3416$, 1578, 1482, 1459, 1259, 1217, 1175, 1031, 796, $780 \mathrm{~cm}^{-1} .{ }^{1} \mathrm{H}-\mathrm{NMR}\left(300 \mathrm{MHz}, \mathrm{DMSO}-d_{6}\right): \delta=2.09$ (qu, $J=6.3 \mathrm{~Hz}, 2 \mathrm{H}, \mathrm{H}-2), 2.41$ (t, $J=6.3 \mathrm{~Hz}, 2 \mathrm{H}, \mathrm{H}-3), 2.92$ (t, $J=6.3 \mathrm{~Hz}, 2 \mathrm{H}, \mathrm{H}-1), 3.76$ (s, 3H, $\mathrm{CH}_{3} \mathrm{O}$ ), 6.77 (dd, $\left.J=8.7,2.7 \mathrm{~Hz}, 1 \mathrm{H}, \mathrm{H}-7\right), 7.28$ (d, $\left.J=8.7 \mathrm{~Hz}, 1 \mathrm{H}, \mathrm{H}-8\right), 7.45$ (d, $\left.J=2.7 \mathrm{~Hz}, 1 \mathrm{H}, \mathrm{H}-5\right)$, 11.74 (br s, $1 \mathrm{H}, \mathrm{NH}){ }^{13} \mathrm{C}-\mathrm{NMR}\left(75.4 \mathrm{MHz}, \mathrm{DMSO}-d_{6}\right): \delta=22.8(\mathrm{C}-1), 23.4(\mathrm{C}-2), 37.7(\mathrm{C}-3), 55.2\left(\mathrm{CH}_{3} \mathrm{O}\right)$, 102.4 (C-5), 111.6 (C-7), 111.7 (C-4a), 112.2 (C-8), 125.2 (C-4b), 130.5 (C-8a), 152.3 (C-9a), 155.1 (C-6), 192.8 (C-4). MS (70 eV): m/z (\%) $215\left(\mathrm{M}^{+}, 2\right), 155$ (37), 153 (100), 127 (12), 125 (35), 90 (23).

6-Methyl-9H-carbazol-4-ol (8). In a threaded ACE glass pressure tube with a sealed Teflon screw cap, under $\mathrm{N}_{2}$ atmosphere, a mixture of $7 \mathbf{a}(0.20 \mathrm{~g}, 1.0 \mathrm{mmol})$ and $\mathrm{Pd} / \mathrm{C}(10 \%)(0.060 \mathrm{~g}, 0.057 \mathrm{mmol})$ in dry $\mathrm{MeOH}(2.5 \mathrm{~mL})$ was stirred at $270{ }^{\circ} \mathrm{C}$ for $48 \mathrm{~h}$. The solvent was removed under vacuum and the residue purified by column chromatography over silica gel (20 g/g of crude, hexane/EtOAc, 80:20), to give $8(0.168 \mathrm{~g}, 85 \%)$ as a white solid. $R_{\mathrm{f}} 0.30$ (hexane/EtOAc, $7: 3$ ); mp 125-126 ${ }^{\circ} \mathrm{C}$. IR (film): $v_{\max }$ 3404, 1615, 1589, 1455, 1341, 1297, 1267, 1047, 803, 752, $724 \mathrm{~cm}^{-1} .{ }^{1} \mathrm{H}-\mathrm{NMR}\left(500 \mathrm{MHz}, \mathrm{CDCl}_{3}\right)$ : $\delta=2.52\left(\mathrm{~s}, 3 \mathrm{H}, \mathrm{CH}_{3}\right), 5.38($ br s, $1 \mathrm{H}, \mathrm{OH}), 6.53(\mathrm{~d}, J=8.5 \mathrm{~Hz}, 1 \mathrm{H}, \mathrm{H}-3), 6.95(\mathrm{~d}, J=8.5 \mathrm{~Hz}, 1 \mathrm{H}, \mathrm{H}-1)$, 7.17-7.22 (m, 2H, H-2, H-7), 7.26 (t, $J=8.5$ Hz, 1H, H-8), 7.89 (br s, 1H, NH), 8.06 (br s, 1H, H-5). ${ }^{13} \mathrm{C}-\mathrm{NMR}\left(125 \mathrm{MHz}, \mathrm{CDCl}_{3}\right): \delta=21.4\left(\mathrm{CH}_{3}\right), 103.3$ (C-1), $104.9(\mathrm{C}-3), 109.7$ (C-8), $111.6(\mathrm{C}-4 \mathrm{a})$, 122.5 (C-4b), 122.7 (C-5), 126.3 (C-2 or C-7), 126.4 (C-7 or C-2), 129.0 (C-6), 137.0 (C-8a), 141.7 (C-9a), 151.8 (C-4). HRMS (EI): $m / z$ [M $\left.\mathrm{M}^{+}\right]$calcd for $\mathrm{C}_{13} \mathrm{H}_{11} \mathrm{NO}$ : 197.0841; found: 197.0844 .

5-Methoxy-3-methyl-9H-carbazole (Glycoborine, Glycrophylamine, 9). A mixture of 8 (0.150 g, $0.76 \mathrm{mmol})$, $\mathrm{MeI}(0.216 \mathrm{~g}, 1.52 \mathrm{mmol})$ and $\mathrm{K}_{2} \mathrm{CO}_{3}(0.157 \mathrm{~g}, 1.14 \mathrm{mmol})$ in dry acetone $(10 \mathrm{~mL})$ was heated to reflux for $2 \mathrm{~h}$. The solvent was removed under vacuum and the residue purified by column chromatography over silica gel (10 g/g of crude, hexane/EtOAc, 95:5), to give $9(0.151 \mathrm{~g}, 94 \%)$ as a 
white solid. $R_{\mathrm{f}} 0.35$ (hexane/EtOAc, 8:2); mp 133-134 ${ }^{\circ} \mathrm{C}$ [Lit. [33] $154-156{ }^{\circ} \mathrm{C}$; [60] 132-134. ${ }^{\circ} \mathrm{C}$; [61] $155-156{ }^{\circ} \mathrm{C}$; [62] $135{ }^{\circ} \mathrm{C}$ ]. IR (KBr): $v_{\max } 3402,1586,1508,1458,1346,1261,1103,804,719 \mathrm{~cm}^{-1}$. ${ }^{1} \mathrm{H}-\mathrm{NMR}\left(500 \mathrm{MHz}, \mathrm{CDCl}_{3}\right): \delta=2.52\left(\mathrm{~s}, 3 \mathrm{H}, \mathrm{CH}_{3}\right), 4.06\left(\mathrm{~s}, 3 \mathrm{H}, \mathrm{CH}_{3} \mathrm{O}\right), 6.64(\mathrm{~d}, J=8.0 \mathrm{~Hz}, 1 \mathrm{H}, \mathrm{H}-6)$, $6.97(\mathrm{~d}, J=8.0 \mathrm{~Hz}, 1 \mathrm{H}, \mathrm{H}-8), 7.18$ (dd, $J=8.0,1.2 \mathrm{~Hz}, 1 \mathrm{H}, \mathrm{H}-2), 7.24$ (d, $J=8.0 \mathrm{~Hz}, 1 \mathrm{H}, \mathrm{H}-1), 7.29$ (t, $J=8.0 \mathrm{~Hz}, 1 \mathrm{H}, \mathrm{H}-7), 8.00$ (br s, $1 \mathrm{H}, \mathrm{NH}), 8.11$ (br s, $1 \mathrm{H}, \mathrm{H}-4) .{ }^{13} \mathrm{C}-\mathrm{NMR}\left(125 \mathrm{MHz}, \mathrm{CDCl}_{3}\right)$ : $\delta=21.4\left(\mathrm{CH}_{3}\right), 55.3\left(\mathrm{CH}_{3} \mathrm{O}\right), 100.1(\mathrm{C}-6), 103.5(\mathrm{C}-8), 109.5(\mathrm{C}-1), 112.4(\mathrm{C}-4 \mathrm{~b}), 122.8(\mathrm{C}-4 \mathrm{a}), 122.9$ (C-4), 126.1 (C-2), 126.4 (C-7), 128.8 (C-3), 136.9 (C-9a), 141.2 (C-8a), 156.2 (C-5). HRMS (EI): $m / z$ $\left[\mathrm{M}^{+}\right]$calcd for $\mathrm{C}_{14} \mathrm{H}_{13} \mathrm{NO}$ : 211.0997; found: 211.0995 .

\section{Conclusions}

In this work, a short and efficient synthetic route for the construction of 2-, 5-, and 7-oxygenated carbazole alkaloids including natural clausine V (1d) is described. As the key steps, this approach includes a palladium(0)-catalyzed aromatization and a palladium(II)-catalyzed cyclization to provide the 2- and 7-oxygenated tricyclic carbazole framework. In the case of the natural 5-oxygenated carbazole glycoborine (glycrophylamine, 9), the palladium-catalyzed sequence was inverted, with cyclization performed before aromatization. The preparation of natural carbazoles clauszoline-K (1f) and clauszoline-L (1g) was also carried out by transformation of carbazole $\mathbf{1 h}$. This methodology is currently being applied to the synthesis of diverse carbazoles, and the results will be reported in due course.

\section{Acknowledgments}

We thank Professors Francisco Delgado and Gerardo Zepeda for their help in spectrometric analyses, and Bruce A. Larsen for reviewing the use of English in the manuscript. J.T. acknowledges SIP/IPN (Grants 20110172, 20120830, and 20130686) and CONACYT (Grants 83446 and 178319) for financial support. R.B., P.A.M., and A.R. thank CONACYT for awarding them graduate scholarships, and SIP/IPN (PIFI) for scholarship complements. J.T. and E.B. are fellows of the EDI-IPN and COFAA-IPN programs.

\section{Conflicts of Interest}

The authors declare no conflict of interest.

\section{References}

1. Knölker, H.J.; Reddy, K.R. Isolation and synthesis of biologically active carbazole alkaloids. Chem. Rev. 2002, 102, 4303-4427.

2. Knölker, H.J.; Reddy, K.R. Chemistry and Biology of Carbazole Alkaloids. In The Alkaloids Chemistry and Biology; Cordell, G.A., Ed.; Academic Press: Amsterdam, The Netherlands, 2008; Volume 65.

3. Schmidt, A.W.; Reddy, K.R.; Knölker, H.J. Occurrence, biogenesis, and synthesis of biologically active carbazole alkaloids. Chem. Rev. 2012, 112, 3193-3328.

4. Knölker, H.J. Occurrence, biological activity, and convergent organometallic synthesis of carbazole alkaloids. Top. Curr. Chem. 2005, 244, 115-148. 
5. Knölker, H.J. Synthesis of biologically active carbazole alkaloids using selective transition-metal-catalyzed coupling reactions. Chem. Lett. 2009, 38, 8-13.

6. Chakraborty, D.P. In Progress in the Chemistry of Organic Natural Products; Herz, W.; Grisebach, H.; Kirby, G.W., Eds.; Springer-Verlag: Wien, Austria, 1977; Volume 34.

7. Chaichantipyuth, C.; Pummangura, S.; Naowsaran, K.; Thanyavuthi, D. Two new bioactive carbazole alkaloids from the rootbark of clausena harmandiana. J. Nat. Prod. 1988, 51, 1285-1288.

8. Wu, T.S.; Huang, S.C.; Wu, P.L.; Teng, C.M. Carbazole alkaloids from Clausena excavata and their biological activity. Phytochemistry 1996, 43, 133-140.

9. Sunthitikawinsakul, A.; Kongkathip, N.; Kongkathip, B.; Phonnakhu, S.; Daly, J.W.; Spande, T.F.; Nimit, Y.; Rochanaruangrai, S. Coumarins and carbazoles from Clausena excavata exhibited antimycobacterial and antifungal activities. Planta Med. 2003, 69, 155-157.

10. Ma, C.; Case, R.J.; Wang, Y.; Zhang, H.J.; Tan, G.T.; Hung, N.V.; Cuong, N.M.; Franzblau, S.G.; Soejarto, D.D.; Fong, H.H.S.; Pauli, G.F. Anti-tuberculosis constituents from the stem bark of Micromelum hirsutum. Planta Med. 2005, 71, 261-267.

11. Meragelman, K.M.; McKee, T.C.; Boyd, M.R. Siamenol, a new carbazole alkaloid from Murraya siamensis. J. Nat. Prod. 2000, 63, 427-428.

12. Kongkathip, B.; Kongkathip, N.; Sunthitikawinsakul, A.; Napaswat, C.; Yoosook, C. Anti-HIV-1 constituents from Clausena excavata: Part II. Carbazoles and a pyranocoumarin. Phytother. Res. 2005, 19, 557-731.

13. Wang, J.; Zheng, Y.; Efferth, T.; Wang, R.; Shen, Y.; Hao, X. Indole and carbazole alkaloids from Glycosmis montana with weak anti-HIV and cytotoxic activities. Phytochemistry 2005, 66, 697-701.

14. Yenjai, C.; Sripontan, S.; Sriprajun, P.; Kittakoop, P.; Jintasirikul, A.; Tanticharoen, M.; Thebtaranonth, Y. Coumarins and carbazoles with antiplasmodial activity from Clausena harmandiana. Planta Med. 2000, 66, 277-279.

15. Shoeb, A.; Anwer, F.; Kapil, R.S.; Popli, S.P. N-Alkylaminocarbazoles as potential anticonvulsant and diuretic agents. J. Med. Chem. 1973, 16, 425-427.

16. Ferris, R.M.; White, H.L.; Tang, F.L.M.; Russell, A.; Harfenist, M. Rimcazole (BW 234U), a novel antipsychotic agent whose mechanism of action cannot be explained by a direct blockade of postsynaptic dopaminergic receptors in brain. Drug Dev. Res. 1986, 9, 171-188.

17. Gilmore, D.L.; Liu; Y.; Matsumoto, R.R. Review of the pharmacological and clinical profile of rimcazole. CNS Drug Rev. 2004, 10, 1-22.

18. Bhattacharyya, P.; Chakraborty, A. Mukonal, a probable biogenetic intermediate of pyranocarbazole alkaloids from Murraya koenigii. Phytochemistry 1984, 23, 471-472.

19. Chakraborty, D.P.; Bhattacharyya, P.; Islam, A.; Roy, M.S. Structure of murrayacinine: A new carbazole alkaloid from Murraya koenigii spreng. Chem. Ind. 1974, 1974, 165-166.

20. Wu, T.S.; Huang, S.C.; Wu, P.L. Carbazole alkaloids from stem bark of Clausena excavata. Phytochemistry 1996, 43, 1427-1429.

21. Ito, C.; Nakagawa, M.; Wu, T.-S.; Furukawa, H. New carbazole alkaloids from Murraya euchrestifolia. Chem. Pharm. Bull. 1991, 39, 2525-2528.

22. Wu, T.S.; Huang, S.C.; Wu, P.L.; Kuoh, C.S. Alkaloidal and other constituents from the root bark of Clausena excavata. Phytochemistry 1999, 52, 523-527.

23. Ito, C.; Katsuno, S.; Ohta, H.; Omura, M.; Kajiura, I.; Furukawa, H. Constituents of Clausena excavate. Isolation and structural elucidation of new carbazole alkaloids. Chem. Pharm. Bull. 1997, 45, 48-52. 
24. Knölker, H.J.; Wolpert, M. Transition metal complexes in organic synthesis. Part 68: Iron-mediated total synthesis of mukonine and mukonidine by oxidative cyclization with air as the oxidizing agent. Tetrahedron 2003, 59, 5317-5322.

25. St. Jean, D.J., Jr.; Poon, S.F.; Schwarzbach, J.L. A tandem cross-coupling/SNAr approach to functionalized carbazoles. Org. Lett. 2007, 9, 4893-4896.

26. Forke, R.; Krahl, M.P.; Däbritz, F.; Jäger, A.; Knölker, H.J. Transition metals in organic synthesis, Part 87: An efficient palladium-catalyzed route to 2-oxygenated and 2,7-dioxygenated carbazole alkaloids - Total synthesis of 2-methoxy-3-methylcarbazole, glycosinine, clausine L, mukonidine, and clausine V. Synlett 2008, 2008, 1870-1876.

27. Forke, R.; Jäger, A.; Knölker, H.J. First total synthesis of clausine L and pityriazole, a metabolite of the human pathogenic yeast Malassezia furfur. Org. Biomol. Chem. 2008, 6, 2481-2483.

28. Watanabe, T.; Oishi, S.; Fujii, N.; Ohno, H. Palladium-catalyzed direct synthesis of carbazoles via one-pot $\mathrm{N}$-arylation and oxidative biaryl coupling: Synthesis and mechanistic study. J. Org. Chem. 2009, 74, 4720-4726.

29. Sridharan, V.; Martín, M.A.; Menéndez, J.C. Acid-free synthesis of carbazoles and carbazolequinones by intramolecular Pd-catalyzed, microwave-assisted oxidative biaryl coupling reactions-Efficient syntheses of murrayafoline A, 2-methoxy-3-methylcarbazole, and glycozolidine. Eur. J. Org. Chem. 2009, 4614-4621.

30. Tsang, W.C.P.; Munday, R.H.; Brasche, G.; Zheng, N.; Buchwald, S.L. Palladium-catalyzed method for the synthesis of carbazoles via tandem $\mathrm{C}-\mathrm{H}$ functionalization and $\mathrm{C}-\mathrm{N}$ bond formation. J. Org. Chem. 2008, 73, 7603-7610.

31. Witulski, B.; Alayrac, C. A highly efficient and flexible synthesis of substituted carbazoles by rhodium-catalyzed inter- and intramolecular alkyne cyclotrimerizations. Angew. Chem. Int. Ed. 2002, 41, 3281-3284.

32. Krahl, M.P.; Jäger, A.; Krause, T.; Knölker, H.J. First total synthesis of the 7-oxygenated carbazole alkaloid clauszoline-K, 3-formyl-7-hydroxycarbazole, clausine $\mathrm{M}$, clausine $\mathrm{N}$ and the anti-HIV active siamenol using a highly efficient palladium-catalyzed approach. Org. Biomol. Chem. 2006, 4, 3215-3219.

33. Kuethe, J.T.; Childers, K.G. Suzuki-miyaura cross-coupling of 2-nitroarenediazonium tetrafluoroborates: Synthesis of unsymmetrical 2-nitrobiphenyls and highly functionalized carbazoles. Adv. Synth. Catal. 2008, 350, 1577-1586.

34. Kataeva, O.; Krahl, M.P.; Knölker, H.J. First total synthesis of the biologically active 2,7dioxygenated tricyclic carbazole alkaloids 7-methoxy- $O$-methylmukonal, clausine H (clauszoline-C), clausine K (clauszoline-J) and clausine O. Org. Biomol. Chem. 2005, 3, 3099-3101.

35. Alcaide, B.; Almendros, P.; Alonso, J.M.; Fernández, I. Carbocyclization versus oxycyclization on the metal-catalyzed reactions of oxyallenyl C3-linked índoles. J. Org. Chem. 2013, 78, 6688-6701.

36. Alcaide, B.; Almendros, P.; Alonso, J.M.; Cembellín, S.; Fernández, I.; Martínez del Campo, T.; Torres, M.R. Iodine recycling via 1,3-migration in iodoindoles under metal catalysis. Chem. Commun. 2013, 49, 7779-7781.

37. Bautista, R.; Jerezano, A.V.; Tamariz, J. Synthetic approach for constructing the 1-oxygenated carbazole core and its application to the preparation of natural alkaloids. Synthesis 2012, 44, 3327-3336.

38. Knölker, H.J.; Fröhner, W. Palladium-catalyzed total synthesis of the antibiotic carbazole alkaloids carbazomycin G and H. J. Chem. Soc. Perkin Trans. 1 1998, 1998, 173-175. 
39. Knölker, H.J.; Reddy, K.R. Indoloquinones, Part 6. First palladium-mediated oxidative cyclization of arylamino-1,2-benzoquinones to carbazole-3,4-quinones-Application to the total synthesis of carbazoquinocin C and ( \pm )-carquinostatin A. Synlett 1999, 1999, 596-598.

40. Knölker, H.J.; O’Sullivan, N. Palladium-promoted synthesis of hydroxy-substituted 5-cyano-5H-benzo[b]carbazole-6,11-diones. Tetrahedron 1994, 50, 10893-10908.

41. Hagelin, H.; Oslob, J.D.; Åkermark, B. Oxygen as oxidant in palladium-catalyzed inter- and intramolecular coupling reactions. Chem. Eur. J. 1999, 5, 2413-2416.

42. Sissouma, D.; Maingot, L.; Collet, S.; Guingant, A. Concise and efficient synthesis of calothrixin B. J. Org. Chem. 2006, 71, 8384-8389.

43. Iida, H.; Yuasa, Y.; Kibayashi, C. A convenient synthesis of $m$-aminophenols by mercury(II) acetate oxidation of 3-amino-2-cyclohexenones. Synthesis 1982, 1982, 471-472.

44. Bautista, R.; Bernal, P.; Montiel, L.E.; Delgado, F.; Tamariz, J. Total synthesis of the natural carbazoles glycozolicine, mukoline, and mukolidine, starting from 4,5-dimethyleneoxazolidin-2ones. Synthesis 2011, 2011, 929-933.

45. Mithani, S.; Weeratunga, G.; Taylor, N.J.; Dmitrienko, G.I. The kinamycins are diazofluorenes and not cyanocarbazoles. J. Am. Chem. Soc. 1994, 116, 2209-2210.

46. Yogo, M.; Ito, C.; Furukawa, H. Synthesis of some carbazolequinone alkaloids and their analogues. Facile palladium-assisted intramolecular ring closure of arylamino-1,4-benzoquinones to carbazole-1,4-quinones. Chem. Pharm. Bull. 1991, 39, 328-334.

47. Saha, C.; Chowdhury, B.K. Carbazoloquinones from Murraya koenigii. Phytochemistry 1998, 48, 363-366.

48. Lin, G.; Zhang, A. The first synthesis of optically pure biscarbazoles and determination of their absolute configurations. Tetrahedron Lett. 1999, 40, 341-344.

49. Molina, P.; Fresneda, P.M.; Almendros, P. Fused carbazoles by tandem aza Wittig/electrocyclic ring closure, preparation of $6 \mathrm{H}$-pyrido[4,3-b]carbazole, $11 \mathrm{H}-$ pyrido[4,3-a]carbazole and 11H-pyrido[3,4-a]carbazole derivatives. Tetrahedron 1993, 49, 1223-1236.

50. Chakraborty, D.P.; Chowdhury, B.K. Synthesis of murrayanine. J. Org. Chem. 1968, 33, 1265-1268.

51. Murakami, Y.; Yokoo, H.; Watanabe, T. New syntheses of murrayaquinone-A via Fischer indolization of 2-sulfonyloxyphenylhydrazone (Fischer indolization and its related compounds. Part 29). Heterocycles 1998, 49, 127-132.

52. Chakraborty, D.P.; Islam, A.; Bhattacharyya, P. Synthesis of murrayanine. J. Org. Chem. 1973, $38,2728-2729$.

53. Murphy, W.S.; Bertrand, M. Bromoquinone-enaminone annulations: Syntheses of murrayaquinone-A and ( \pm )-bismurrayaquinone-A. J. Chem. Soc. Perkin Trans. 1 1998, 1998, 4115-4119.

54. Crum, J.D.; Sprague, P.W. The synthesis of murrayanine. Chem. Commun. 1966, 1966, 417-418.

55. Sissouma, D.; Collet, S.C.; Guingant, A.Y. A synthesis of calothrixin B. Synlett 2004, 2004, 2612-2614.

56. Forke, R.; Krahl, M.P.; Krause, T.; Schlechtingen, G.; Knölker, H.J. Transition metals in organic synthesis, Part 82. First total synthesis of methyl 6-methoxycarbazole-3-carboxylate, glycomaurrol, the anti-TB active micromeline, and the furo[2,3-c]carbazole alkaloid eustifoline-D. Synlett 2007, 2007, 268-272. 
57. Schmidt, M.; Knölker, H.J. Transition metals in organic synthesis, Part 91: Palladium-catalyzed approach to 2,6-dioxygenated carbazole alkaloids-First total synthesis of the phytoalexin carbalexin C. Synlett 2009, 2009, 2421-2424.

58. Knölker, H.J.; Goesmann, H.; Hofmann, C. Transition metal complexes in organic synthesis, Part 31. A novel molybdenum-mediated synthesis of carbazole derivatives: Application to the total synthesis of mukonal and 1,1'-bis(2-hydroxy-3-methylcarbazole). Synlett 1996, 1996, 737-740.

59. Roy, S.; Bhattacharyya, L.; Chakraborty, D.P. Structure and synthesis of mukoline and mukolidine, two new carbazole alkaloids from Murraya koenigii spreng. J. Indian Chem. Soc. 1982, 59, 1369-1371.

60. Cheenpracha, S.; Laphookhieo, S. Alkaloids and amides from Glycosmis macrophylla. Phytochem. Lett. 2011, 4, 187-189.

61. Chakravarty, A.K.; Sarkar, T.; Masuda, K.; Takey, T.; Doi, H.; Kotani, E.; Shiojima, K. Structure and synthesis of glycoborine, a new carbazole alkaloid from the roots of Glycosmis arborea: A note on the structure of glycozolicine. Indian J. Chem. 2001, 40B, 484-489.

62. Jash, S.S.; Biswas, G.K.; Bhattacharyya, S.K.; Bhattacharyya, P.; Chakraborty. A.; Chowdhury, B.K. Carbazole alkaloids from Glycosmis pentaphylla. Phytochemistry 1992, 31, 2503-2505.

63. Makawana, J.A.; Patel, M.P.; Patel, R.G. Synthesis and in vitro antimicrobial activity of $\mathrm{N}$-arylquinoline derivatives bearing 2-morpholinoquinoline moiety. Chinese Chem. Lett. 2012, 23, 427-430.

64. Iida, H.; Yuasa, Y.; Kibayashi, C. Intramolecular cyclization of enaminones involving arylpalladium complexes. Synthesis of carbazoles. J. Org. Chem. 1980, 45, 2938-2942.

65. Andrade, C.K.Z.; Barreto, A.F.S.; Wender, A.S. Microwave assisted solvent-, support- and catalyst-free synthesis of enaminones. Arkivoc 2008, xii, 226-232.

66. Maiti, D.; Buchwald, S.L. Orthogonal $\mathrm{Cu}-$ and Pd-based catalyst systems for the $O$ - and $N$-arylation of aminophenols. J. Am. Chem. Soc. 2009, 131, 17423-17429.

67. Urgaonkar, S.; Xu, J.H.; Verkade, J.G. Application of a new bicyclic triaminophosphine ligand in Pd-catalyzed Buchwald-Hartwig amination reactions of aryl chlorides, bromides, and iodines. J. Org. Chem. 2003, 68, 8416-8423.

68. Bhatia, S.K.; Kapil, R.S. Synthesis of $O$-methylcycloclausanitin. Indian J. Chem. Soc. 1984, 23B, 296-299.

69. Oikawa, Y.; Yonemitsu, O. Selective oxidation of the side chain at C-3 of indoles. J. Org. Chem. 1977, 42, 1213-1216.

70. Weng, B.; Liu, R.; Li, J.-H. An improved method for the synthesis of carbazolones by palladium/copper-catalyzed intramolecular annulation of $\mathrm{N}$-arylenaminones. Synthesis 2010, 2010, 2926-2930.

Sample Availability: Samples of the compounds $4 \mathbf{a}-\mathbf{e}, \mathbf{5 a}, \mathbf{5 d}, \mathbf{5 e}, \mathbf{1 d}, \mathbf{1 f}-\mathbf{g}, \mathbf{1 h}-\mathbf{i}$, and $\mathbf{1 k}$ are available from the authors.

(C) 2013 by the authors; licensee MDPI, Basel, Switzerland. This article is an open access article distributed under the terms and conditions of the Creative Commons Attribution license (http://creativecommons.org/licenses/by/3.0/). 